\title{
The ACS Fornax Cluster Survey. III. Globular Cluster Specific Frequencies of Early-type Galaxies
}

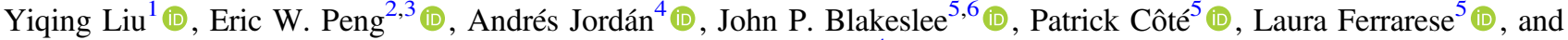 \\ Thomas H. Puzia ${ }^{4}$ (D) \\ ${ }^{1}$ Sub-department of Astrophysics, Department of Physics, University of Oxford, Denys Wilkinson Building, Keble Road, Oxford OX1 3RH, UK \\ yiqing.liu@physics.ox.ac.uk, halfxianr@gmail.com \\ ${ }^{2}$ Department of Astronomy, Peking University, Beijing 100871, People's Republic of China; peng@pku.edu.cn \\ ${ }^{3}$ Kavli Institute for Astronomy and Astrophysics, Peking University, Beijing 100871, People's Republic of China \\ ${ }^{4}$ Instituto de Astrofísica, Facultad de Física, Pontificia Universidad Católica de Chile, Av. Vicuña Mackenna 4860, 7820436 Macul, Santiago, Chile \\ ${ }^{5}$ National Research Council of Canada, Herzberg Astronomy and Astrophysics Research Centre, 5071 W. Saanich Road, Victoria, BC V9E 2E7, Canada \\ ${ }^{6}$ Gemini Observatory, Casilla 603, La Serena, Chile \\ Received 2018 August 3; revised 2019 March 13; accepted 2019 March 22; published 2019 April 25
}

\begin{abstract}
The globular cluster (GC) specific frequency $\left(S_{N}\right)$, defined as the number of GCs per unit galactic luminosity, represents the efficiency of GC formation (and survival) compared to field stars. Despite the naive expectation that star cluster formation should scale directly with star formation, this efficiency varies widely across galaxies. To explore this variation, we measure the $z$-band GC specific frequency $\left(S_{N, z}\right)$ for 43 early-type galaxies (ETGs) from the Hubble Space Telescope (HST)/Advanced Camera for Surveys (ACS) Fornax Cluster Survey. Combined with the homogeneous measurements of $S_{N, z}$ in 100 ETGs from the HST/ACS Virgo Cluster Survey from Peng et al., we investigate the dependence of $S_{N, z}$ on mass and environment over a range of galaxy properties. We find that $S_{N, z}$ behaves similarly in the two galaxy clusters, despite the clusters' order-of-magnitude difference in mass density. The $S_{N, z}$ is low in intermediate-mass ETGs $\left(-20<M_{z}<-23\right)$ and increases with galaxy luminosity. It is elevated at low masses, on average, but with a large scatter driven by galaxies in dense environments. The densest environments with the strongest tidal forces appear to strip the GC systems of low-mass galaxies. However, in low-mass galaxies that are not in strong tidal fields, denser environments correlate with enhanced GC formation efficiencies. Normalizing by inferred halo masses, the GC mass fraction, $\eta=(3.36 \pm 0.2) \times 10^{-5}$, is constant for ETGs with stellar masses $\mathcal{M}_{\star} \lesssim 3 \times 10^{10} M_{\odot}$, in agreement with previous studies. The lack of correlation between the fraction of GCs and the nuclear light implies only a weak link between the infall of GCs and the formation of nuclei.
\end{abstract}

Key words: galaxies: clusters: individual (Virgo, Fornax) - galaxies: star clusters: general

\section{Introduction}

Globular clusters (GCs) are old, massive, compact star clusters whose masses range from $10^{4}$ to $10^{6} M_{\odot}$, with a typical size of $\sim 3$ pc. They are mostly associated with the old spheroid components of galaxies, with the oldest GCs having ages comparable to the universe.

Although the exact formation scenario for GCs is still unknown, it is believed that they form in high-pressure and high-density regions with high star formation efficiencies on short timescales. In such environments, they might gain enough mass before feedback processes halt star formation (e.g., Elmegreen \& Efremov 1997; Kruijssen 2012). One possibility is that GCs are the merger remnants of protogalactic disk fragments in the minihalos at high redshift. Kimm et al. (2016) simulated this scenario in a cosmological context and found that it reproduced well the basic GC properties of mass, size, and star formation history.

In the local universe, young massive star clusters form in regions with high star formation rate surface densities (Larsen \& Richtler 2000), and evidence shows that they might be the progenitors of GCs (e.g., Whitmore et al. 1999). It is likely that GCs also form during violent star formation events, which could happen at high redshift or in gas-rich galactic mergers. However they are formed, studying GC systems provides a unique window onto the earliest and most intense episodes of galactic star formation. We can also learn about the subsequent galactic mass assembly histories, in which a majority of GCs in massive galaxies are likely accreted from less massive galaxies in a hierarchical fashion (Côté et al. 1998; Tonini 2013). At the same time, such studies provide information about the formation mechanisms of massive star clusters.

While the existence of GC systems beyond the Local Group has been known for many decades (Baum 1955), the advent of CCDs and the Hubble Space Telescope (HST) led to the first systematic studies of extragalactic GC systems-their numbers, sizes, luminosities, colors, and stellar populations-as a function of host galaxy properties (e.g., Larsen et al. 2001; Jordán et al. 2005, 2007b; Peng et al. 2006, 2008; Georgiev et al. 2010; Masters et al. 2010). Review papers by Harris (1991) and Brodie \& Strader (2006) tracked the progress of research in the field of extragalactic GC systems and summarized the state of the art at their respective times.

The number of GCs in a galaxy is one of the most basic observables of a GC system. While the number of GCs broadly correlates with galaxy stellar mass, as one might expect, the ratio of the number of GCs to the stellar luminosity is famously not constant. This ratio is widely parameterized as the GC specific frequency $\left(S_{N}\right)$-defined as the number of GCs per unit of the galactic $V$-band luminosity and normalized to $M_{V}=-15$ (Harris \& van den Bergh 1981) — and represents the efficiency of GC formation (and survival) with respect to field stars.

Previous studies have measured $S_{N}$ in galaxies with a wide range of properties. One of the largest individual such studies, and the one most relevant to this paper, is that of Peng et al. 
(2008), which used the data from the ACS Virgo Cluster Survey (ACSVCS; Côté et al. 2004; Jordán et al. 2009) to study $S_{N}$ in 100 Virgo cluster early-type galaxies (ETGs) over a wide range of galactic mass. The Virgo cluster ETGs were found to have a "U-shaped" relation between $S_{N}$ and galactic luminosity, a shape that they showed mimicked the effects of the stellar mass-halo mass relation of galaxies determined from halo abundance matching. It was found that $S_{N}$, or GC mass fractions, were universally low in intermediate-mass galaxies $\left(-20.5<M_{V}<-18\right)$ but with upturns occurring at both high and low mass. Other studies using independent data sets show similar results for ETGs (e.g., Zaritsky et al. 2015).

Because GCs do not form in a constant fraction out of field stars, GC formation might be governed by more fundamental regulators. Blakeslee (1997, 1999) and Blakeslee et al. (1997) found that the number of GCs of the bright central galaxies (BCGs) scaled with the total mass of entire galaxy clusters. Furthermore, McLaughlin (1999) found that in massive ETGs, the data are consistent with a universal fraction of the baryonic mass ending up in GCs. Later studies show correlations between GC number and the halo mass of individual galaxies (Spitler \& Forbes 2009; Georgiev et al. 2010; Harris et al. 2013; Hudson et al. 2014). This is also supported by simulations (Kravtsov \& Gnedin 2005). These works indicate that the relation between $S_{N}$ and galactic luminosity might come from the similarly shaped distribution of the galactic halo mass-to-light ratio $\left(M_{h} / L\right)$ with galactic mass (Wolf et al. 2010).

Traditionally, studies of $S_{N}$ focus on its relationship with galactic masses or types. Recently, however, the role of environment has turned out to be important, especially at low masses. Peng et al. (2008) showed that while $S_{N}$ is systematically higher for both high- and low-mass ETGs, it has a large scatter in the low-mass range. Peng et al. (2008) studied this scatter in low-mass ETG $S_{N}$, finding that galaxies closer to the cluster center have higher $S_{N}$ and suggesting that their $S_{N}$ are regulated by their environment.

There are two possible (and nonexclusive) explanations for such an environmental dependence. One is that the low-mass galaxies in denser regions had stronger star formation at an early time, and more GCs formed along with it. Alternatively, the star formation of low-mass galaxies stopped earlier in denser environments, and because most GCs formed at very early times, the number of GCs is hardly affected. The decrease of the number of field stars, however, drives up $S_{N}$ in dense environments. Both scenarios are supported by the higher $[\alpha / \mathrm{Fe}]$ at smaller clustercentric distances from observations (Liu et al. 2016b).

Nonetheless, more evidence is needed to expand our understanding of this apparent environmental dependence of $S_{N}$. The correlation shown in Peng et al. (2008) is driven by the high $S_{N}$ of the innermost galaxies, and no clear dependence is found in the lower-mass sample of Miller \& Lotz (2007). The environmental dependence of $[\alpha / \mathrm{Fe}]$ is similarly tantalizing. Liu et al. (2016a) found that only the densest environments affect the $[\alpha / \mathrm{Fe}]$ distribution of low-mass ETGs.

Therefore, the importance of environment for $\mathrm{GC}$ formation is a question. A place to study environmental effects is galaxy clusters, as the mass density changes with clustercentric distance. In addition, comparing galaxy clusters with different densities provides further constraints. Peng et al. (2008) studied the $S_{N}$ of ETGs and their relations with the masses and environments in the nearest galaxy cluster, and we extend this work by adding samples from the next-nearest galaxy cluster.
As a complementary program to the ACSVCS, the ACS Fornax Cluster Survey (ACSFCS; Jordán et al. 2007a) imaged 43 galaxies in the Fornax Cluster, a galaxy cluster that, compared to Virgo, is $\sim 20 \%$ more distant (Blakeslee et al. 2009), an order of magnitude less massive, and dynamically older. Because they have similar virial radii, the mass density of Fornax is significantly lower. In this work, we measure the $S_{N}$ of 43 ETGs from the ACSFCS sample. Combining with the results of 100 ETGs from the ACSVCS sample, which were measured by Peng et al. (2008), we study the dependence of $S_{N}$ on mass and environment among ETGs with a significant sample size.

The paper is structured as follows. Our data are introduced in Section 2. Section 3 describes our measurement of the number of GCs. We then present the relationships between $S_{N}$, galactic masses, and environments in Section 4 and a related discussion in Section 5. In Section 6, we list our main conclusions.

\section{Observations and Data}

The ACSFCS program imaged 43 ETGs in the Fornax Cluster with the HST Advanced Camera for Surveys (ACS; Ford et al. 1998). This is a complete sample of the Fornax galaxies that are brighter than $B_{T} \sim 15.5\left(M_{B} \sim-16\right) \mathrm{mag}$, covering the morphological types of E, S0, SB0, dE, dE,N, $\mathrm{dS} 0$, and $\mathrm{dS} 0, \mathrm{~N}$. It includes 41 galaxies from the Fornax Cluster Catalog (FCC; Ferguson 1989) and two outlying elliptical galaxies, NGC 1340 and IC 2006.

Each galaxy was imaged in two HST/ACS filters, F475W and F850LP. The field of view (FOV) is $202^{\prime \prime} \times 202^{\prime \prime}$, with a pixel scale of $0 . \prime 049$. The two filters are roughly the same as the Sloan Digital Sky Survey (SDSS) $g$ and $z$ bands. Hereafter, they are referred as the $g$ or $z$ bands. For a full description of the ACSFCS and its data, we refer the reader to the survey description paper (Jordán et al. 2007a) and the source catalog paper (Jordán et al. 2015). The basic properties of these 43 ETGs are listed in Table 1. The galactic magnitudes are derived from the integration of the best-fit Sérsic luminosity radial profiles, similar to the work of Ferrarese et al. (2006) for the ACSVCS samples. The stellar masses are from the estimates of $M_{\star} / L_{z}$ using the $g-z$ color and the models of Bruzual \& Charlot (2003), assuming a single age of 10 Gyr. These are the same galaxy quantities used in Turner et al. (2012).

For the purpose of studying GCs, the images are sufficiently deep so that $\sim 90 \%$ of the GCs within the ACS FOV can be detected (Côté et al. 2004). We also used 16 blank high-latitude control fields with simulated Fornax galaxies introduced into the data to estimate the field-dependent contamination of background galaxies (e.g., Peng et al. 2006).

Jordán et al. (2004) described the image analysis and pointsource selection. Jordán et al. $(2009,2015)$ provided the GC catalogs with detection probabilities $\left(p_{\mathrm{GC}}\right)$ for the ACSVCS and ACSFCS data, respectively. The parameter $p_{\mathrm{GC}}$, the probability of an object being a GC, is a function of an object's apparent magnitude $(m)$, projected half-light radius $\left(r_{h}\right)$, and flux of the local background $\left(I_{b}\right)$. Following the previous ACSVCS and ACSFCS papers, we select GCs by requiring $p_{\mathrm{GC}} \geqslant 0.5$.

In our $S_{N}$ analysis, we will combine these data with the measurements from the ACSVCS sample (Peng et al. 2008). The ACSVCS observed 100 ETGs in the Virgo Cluster with $B_{T}<16$, but the sample is only complete to $B_{T}<12.15$ $\left(M_{B}<-18.94\right)$. It has an instrument setup identical to the 
Table 1

Global Properties of the Galaxies in ACSFCS

\begin{tabular}{|c|c|c|c|c|c|}
\hline $\begin{array}{l}\text { Name } \\
\text { (1) }\end{array}$ & $\begin{array}{l}\text { Other } \\
(2)\end{array}$ & $\begin{array}{c}M_{V} \\
(3)\end{array}$ & $\begin{array}{l}M_{z} \\
(4)\end{array}$ & $\begin{array}{l}R_{p} \\
(5)\end{array}$ & $\begin{array}{c}M_{\star} \\
(6)\end{array}$ \\
\hline FCC 21 & NGC 1316 & -23.78 & -24.45 & 1.27 & 758.64 \\
\hline FCC 213 & NGC 1399 & -22.77 & -23.49 & 0.00 & 263.02 \\
\hline FCC 219 & NGC 1404 & -21.73 & -22.52 & 0.05 & 139.63 \\
\hline NGC 1340 & ESO 418-G005 & -21.59 & -22.22 & 1.69 & 97.76 \\
\hline FCC 167 & NGC 1380 & -21.83 & -22.46 & 0.21 & 61.27 \\
\hline FCC 276 & NGC 1427 & -20.99 & -21.68 & 0.27 & 46.79 \\
\hline FCC 147 & NGC 1374 & -20.79 & -21.47 & 0.24 & 49.21 \\
\hline IC 2006 & ESO 359-G007 & -20.00 & -20.75 & 1.15 & 24.14 \\
\hline FCC 83 & NGC 1351 & -20.50 & -21.18 & 0.59 & 32.13 \\
\hline FCC 184 & NGC 1387 & -20.95 & -21.75 & 0.11 & 65.49 \\
\hline FCC 63 & NGC 1339 & -19.98 & -20.75 & 1.32 & 26.76 \\
\hline FCC 193 & NGC 1389 & -20.26 & -20.90 & 0.13 & 30.37 \\
\hline FCC 153 & IC 1963 & -19.06 & -19.74 & 0.40 & 8.68 \\
\hline FCC 170 & NGC 1381 & -19.94 & -20.63 & 0.14 & 19.70 \\
\hline FCC 177 & NGC 1380A & -19.02 & -19.70 & 0.27 & 6.67 \\
\hline FCC 47 & NGC 1336 & -19.48 & -20.14 & 0.85 & 9.33 \\
\hline FCC 43 & IC 1919 & -18.94 & -19.51 & 1.25 & 2.99 \\
\hline FCC 190 & NGC $1380 B$ & -18.92 & -19.62 & 0.13 & 7.45 \\
\hline FCC 310 & NGC 1460 & -19.10 & -19.75 & 0.70 & 8.92 \\
\hline FCC 148 & NGC 1375 & -19.35 & -19.92 & 0.23 & 10.77 \\
\hline FCC 249 & NGC 1419 & -19.13 & -19.77 & 0.73 & 9.82 \\
\hline FCC 255 & ESO 358-G50 & -18.71 & -19.32 & 0.61 & 3.59 \\
\hline FCC 277 & NGC 1428 & -18.82 & -19.47 & 0.29 & 7.55 \\
\hline FCC 55 & ESO 358-G06 & -18.34 & -18.98 & 0.85 & 4.00 \\
\hline FCC 152 & ESO 358-G25 & -18.42 & -18.96 & 1.06 & 3.49 \\
\hline FCC 301 & ESO 358-G59 & -18.46 & -19.08 & 0.50 & 4.51 \\
\hline FCC 335 & ESO 359-G02 & -17.81 & -18.36 & 0.87 & 1.27 \\
\hline FCC 143 & NGC 1373 & -18.50 & -19.16 & 0.26 & 5.11 \\
\hline FCC 95 & PGC 13084 & -17.90 & -18.52 & 0.50 & 2.76 \\
\hline FCC 136 & PGC 13230 & -17.56 & -18.15 & 0.28 & 1.96 \\
\hline FCC 182 & PGC 13343 & -17.71 & -18.37 & 0.11 & 2.49 \\
\hline FCC 204 & ESO 358-G43 & -17.55 & -18.14 & 0.81 & 1.80 \\
\hline FCC 119 & PGC 13177 & -17.21 & -17.79 & 0.74 & 1.06 \\
\hline FCC 26 & ESO 357-G25 & -17.32 & -17.54 & 1.06 & 0.25 \\
\hline FCC 90 & PGC 13058 & -17.44 & -17.81 & 0.59 & 1.20 \\
\hline FCC 106 & PGC 13146 & -17.38 & -17.96 & 0.58 & 1.44 \\
\hline FCC 19 & ESO 301-G08 & -16.89 & -17.40 & 1.33 & 0.34 \\
\hline FCC 288 & ESO 358-G56 & -16.92 & -17.47 & 0.63 & 0.65 \\
\hline FCC 202 & NGC 1396 & -17.12 & -17.68 & 0.02 & 1.08 \\
\hline FCC 324 & ESO 358-G66 & -16.90 & -17.47 & 0.75 & 0.57 \\
\hline FCC 100 & PGC 13097 & -16.80 & -17.28 & 0.49 & 0.86 \\
\hline FCC 203 & ESO 358-G42 & -16.97 & -17.52 & 0.32 & 0.93 \\
\hline FCC 303 & PGC 13758 & -17.34 & -17.88 & 0.70 & 0.52 \\
\hline
\end{tabular}

Note. Column (1): FCC or primary designation. Column (2): alternate name. Columns (3) and (4): absolute $V$ and $z$ magnitude, adopted from the unpublished measurements used in Turner et al. (2012). Column (5): projected distance from FCC 213 (NGC 1399) in Mpc. Column (6): stellar mass $\left(10^{9} M_{\odot}\right)$, adopted from the measurements used in Turner et al. (2012).

ACSFCS, with data reduction and GC selection performed in the exact same way.

\section{Counting GCs}

\subsection{Total Numbers of GCs}

The stellar light of the galaxy creates a spatially varying completeness limit specific to each galaxy. Similar to the ACSVCS, most of the galaxies in the sample have lower mass and thus low surface brightness and smaller spatial extent. For these galaxies, we find that a simple counting of GCs, corrected for contamination and the faint end of the GC luminosity function (GCLF), is sufficient. In each galaxy, we select GC candidates that are brighter than $1 \sigma$ above the mean of its GCLF (previously measured using the same data by Villegas et al. 2010). These are bright enough to be detected regardless of the underlying surface brightness of the galaxy. To estimate the background contamination, we perform the same selection in control fields. Because the typical number of contaminants is less than 10 and their spatial distributions are nearly uniform, we use the same background correction at all galactocentric distances. The average density and $1 \sigma$ uncertainty of the contaminants are derived by the mean number and standard deviation of the selected candidates from 16 control fields.

To count the total number of GCs, we order the selected GCs in a galaxy by their galactocentric distances. From the center to the outskirts, we add the number of $\left(1-n_{B} * d A i\right) / p_{\mathrm{GC} i}$ at each (the $i$ th) $\mathrm{GC}$, where $n_{B}, d A i$, and $p_{\mathrm{GCi}}$ are the mean density of contaminants, the area of the annulus between the $(i-1)$ th and $i$ th GC, and the $p_{\mathrm{GC}}$ of this GC, respectively. For the low-mass ETGs $\left(M_{z}>-20\right)$, the differential number densities at the outermost GCs are close to zero. Therefore, the total number of GCs in low-mass Fornax ETGs are just the cumulative numbers at the outermost GCs. For the massive ETGs $\left(M_{z} \leqslant-20\right)$, we fit cumulative Sérsic radial profiles and extrapolate them to infinity to derive the total numbers. For FCC 170 and FCC 193, because the results from profile fitting and simple sum are consistent with each other, we adopt the number from simple sum. The results of profile fitting and GC numbers are listed in Tables 2 and 3.

For the ACSVCS sample, we simply adopt the measurements published in Peng et al. (2008). In their work, the GC number of low-mass ETGs $\left(M_{z}>-19\right)$ is counted by summing up the number of GCs, using a representative completeness limit for each galaxy, subtracting the contamination estimated by the control fields, and correcting for faint GCs using the GCLFs measured by Jordán et al. (2007b), which is essentially the same technique as what we have done for the ACSFCS galaxies. For the massive Virgo ETGs, Peng et al. (2008) fit the binned radial density profiles rather than the cumulative profiles, as we do here.

We note that Harris et al. (2013) published GC numbers for most of our ACSFCS samples. Their numbers were adopted from Villegas et al. (2010) without corrections for either background contamination or completeness. Although the difference between most of our estimates and the Villegas et al. (2010) numbers is similar to the uncertainty, the numbers for low-mass ETGs presented in Harris et al. (2013) are systematically higher than ours. The mean difference is 10.21, which matches the expected contaminant numbers. Additionally, our more accurate measurements of the two most massive galaxies, FCC 213 and FCC 21, are significantly higher than that due to our accounting for GCs not in the ACS FOV. Bassino et al. (2006) and Richtler et al. (2014) took wide-field images of the massive ETGs FCC 21, FCC 147, and FCC 184 and measured the radial density profiles of their GC systems. Our measurements are consistent with theirs within the uncertainties.

\subsubsection{Special Cases}

Below, we describe some atypical or special cases.

FCC 213 (NGC 1399). A counterpart of M87 in Virgo, FCC 213 is the BCG of the Fornax Cluster. The FOV is insufficient to encompass its entire GC system, so we need to 
Table 2

The Cumulative Sérsic Profile Fitting Results of the GC Systems in ACSFCS Galaxies

\begin{tabular}{|c|c|c|c|c|c|c|c|c|c|}
\hline $\begin{array}{l}\text { ID } \\
\text { (1) }\end{array}$ & $\begin{array}{l}N_{e, \text { tot }} \\
(2)\end{array}$ & $\begin{array}{c}R_{e, \text { tot }}(\operatorname{arcsec}) \\
\text { (3) }\end{array}$ & $\begin{array}{l}n_{\text {tot }} \\
(4)\end{array}$ & $\begin{array}{l}N_{e, \text { blue }} \\
\text { (5) }\end{array}$ & $\begin{array}{c}R_{e, \text { blue }}(\operatorname{arcsec}) \\
\text { (6) }\end{array}$ & $\begin{array}{c}n_{\text {blue }} \\
(7)\end{array}$ & $\begin{array}{c}N_{e, \text { red }} \\
\text { (8) }\end{array}$ & $\begin{array}{c}R_{e, \text { red }}(\operatorname{arcsec}) \\
\quad \text { (9) }\end{array}$ & $\begin{array}{l}n_{\text {red }} \\
(10)\end{array}$ \\
\hline $\bar{F} 21$ & $0.0343 \pm 0.0007$ & $77.87 \pm 1.99$ & $0.40 \pm 0.01$ & $0.0189 \pm 0.0013$ & $93.82 \pm 15.10$ & $0.41 \pm 0.04$ & $0.0086 \pm 0.0022$ & $107.49 \pm 27.10$ & $0.82 \pm 0.18$ \\
\hline F213 & $0.0010 \pm 0.0018$ & $655.78 \pm 233.43$ & $3.34 \pm 0.47$ & $0.0004 \pm 0.0004$ & $730.56 \pm 277.39$ & $2.88 \pm 0.67$ & $0.0011 \pm 0.0012$ & $551.21 \pm 163.73$ & $2.59 \pm 0.31$ \\
\hline N1340 & $0.0082 \pm 0.0009$ & $56.91 \pm 3.91$ & $1.21 \pm 0.10$ & $0.0037 \pm 0.0008$ & $83.73 \pm 14.26$ & $1.63 \pm 0.22$ & $0.0036 \pm 0.0009$ & $37.88 \pm 6.47$ & $0.85 \pm 0.31$ \\
\hline F219 & $0.0160 \pm 0.0008$ & $42.45 \pm 1.18$ & $0.76 \pm 0.05$ & $0.0065 \pm 0.0011$ & $46.62 \pm 15.27$ & $0.64 \pm 0.24$ & $0.0099 \pm 0.0010$ & $39.24 \pm 2.12$ & $0.85 \pm 0.10$ \\
\hline F167 & $0.0149 \pm 0.0019$ & $53.45 \pm 9.74$ & $0.97 \pm 0.16$ & $0.0058 \pm 0.0007$ & $44.70 \pm 3.38$ & $0.83 \pm 0.12$ & $0.0081 \pm 0.0014$ & $63.18 \pm 15.19$ & $1.15 \pm 0.20$ \\
\hline F276 & $0.0117 \pm 0.0014$ & $52.25 \pm 6.23$ & $1.35 \pm 0.15$ & $0.0044 \pm 0.0007$ & $74.94 \pm 9.56$ & $1.47 \pm 0.15$ & $0.0079 \pm 0.0012$ & $37.14 \pm 3.20$ & $1.26 \pm 0.16$ \\
\hline F147 & $0.0073 \pm 0.0011$ & $67.48 \pm 7.03$ & $1.48 \pm 0.14$ & $0.0050 \pm 0.0008$ & $58.40 \pm 6.67$ & $0.99 \pm 0.14$ & $\cdots$ & $\ldots$ & $\cdots$ \\
\hline I2006 & $0.0111 \pm 0.0013$ & $30.58 \pm 1.89$ & $0.93 \pm 0.13$ & $0.0023 \pm 0.0012$ & $54.08 \pm 32.06$ & $2.18 \pm 0.77$ & $0.0079 \pm 0.0012$ & $29.14 \pm 2.74$ & $0.61 \pm 0.16$ \\
\hline F83 & $0.0137 \pm 0.0018$ & $36.93 \pm 2.46$ & $1.88 \pm 0.14$ & $0.0048 \pm 0.0014$ & $51.68 \pm 9.53$ & $2.55 \pm 0.36$ & $0.0077 \pm 0.0022$ & $33.21 \pm 11.81$ & $1.59 \pm 0.48$ \\
\hline F184 & $0.0131 \pm 0.0006$ & $51.39 \pm 4.29$ & $0.40 \pm 0.04$ & $0.0044 \pm 0.0004$ & $43.60 \pm 5.84$ & $0.47 \pm 0.10$ & $0.0085 \pm 0.0004$ & $56.22 \pm 6.91$ & $0.40 \pm 0.05$ \\
\hline F63 & $0.0177 \pm 0.0012$ & $32.73 \pm 1.15$ & $1.03 \pm 0.07$ & $0.0084 \pm 0.0015$ & $41.07 \pm 11.77$ & $0.95 \pm 0.28$ & $0.0112 \pm 0.0017$ & $24.60 \pm 2.02$ & $1.11 \pm 0.19$ \\
\hline F47 & $0.0118 \pm 0.0014$ & $40.57 \pm 2.59$ & $1.58 \pm 0.13$ & $\ldots$ & & $\ldots$ & $0.0168 \pm 0.0027$ & $20.25 \pm 2.52$ & $0.89 \pm 0.23$ \\
\hline
\end{tabular}

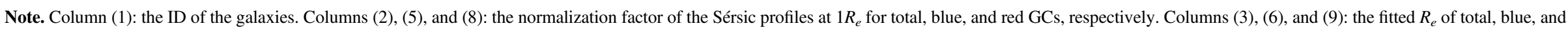
red GCs. Columns (4), (7), and (10): the Sérsic index of total, blue, and red GCs. 
Table 3

The Numbers and Formation Efficiencies of GCs in ACSFCS Galaxies

\begin{tabular}{|c|c|c|c|c|c|c|c|}
\hline $\begin{array}{l}\text { ID } \\
\text { (1) }\end{array}$ & $\begin{array}{c}N_{\mathrm{GC}} \\
(2)\end{array}$ & $\begin{array}{l}N_{\mathrm{GC}, \text { blue }} \\
\text { (3) }\end{array}$ & $\begin{array}{c}N_{\mathrm{GC}, \text { red }} \\
\text { (4) }\end{array}$ & $\begin{array}{l}S_{N} \\
(5)\end{array}$ & $\begin{array}{c}S_{N, z} \\
(6)\end{array}$ & $\begin{array}{c}T \\
(7)\end{array}$ & $\begin{array}{l}f_{\text {red }} \\
(8)\end{array}$ \\
\hline FCC 21 & $2541.66 \pm 514.16$ & $1442.2 \pm 406.5$ & $1099.49 \pm 314.83$ & $0.54 \pm 0.02$ & $0.29 \pm 0.01$ & $2.35 \pm 1.03$ & $0.19 \pm 0.23$ \\
\hline FCC 213 & $9693.0 \pm 3263.3$ & $4234.8 \pm 813.7$ & $6655.4 \pm 1841.3$ & $7.49 \pm 2.52$ & $3.87 \pm 1.30$ & $36.85 \pm 23.59$ & $0.61 \pm 0.20$ \\
\hline FCC 219 & $310.6 \pm 8.16$ & $141.7 \pm 37.2$ & $171.7 \pm 8.3$ & $0.62 \pm 0.01$ & $0.30 \pm 0.00$ & $2.22 \pm 0.88$ & $0.54 \pm 0.07$ \\
\hline FCC 167 & $503.9 \pm 76.6$ & $129.5 \pm 9.0$ & $374.3 \pm 77.2$ & $0.92 \pm 0.14$ & $0.52 \pm 0.07$ & $8.22 \pm 1.25$ & $0.74 \pm 0.19$ \\
\hline FCC 276 & $440.4 \pm 39.7$ & $295.5 \pm 40.9$ & $145.0 \pm 10.0$ & $1.76 \pm 0.15$ & $0.93 \pm 0.08$ & $9.41 \pm 5.20$ & $0.32 \pm 0.03$ \\
\hline FCC 147 & $473.7 \pm 41.8$ & $206.8 \pm 22.6$ & $266.9 \pm 47.5$ & $2.26 \pm 0.20$ & $1.22 \pm 0.10$ & $9.62 \pm 4.12$ & $0.56 \pm 0.11$ \\
\hline FCC 83 & $298.1 \pm 13.6$ & $172.4 \pm 26.4$ & $125.7 \pm 22.6$ & $1.87 \pm 0.08$ & $1.00 \pm 0.04$ & $9.27 \pm 4.71$ & $0.42 \pm 0.07$ \\
\hline FCC 184 & $299.0 \pm 30.9$ & $75.6 \pm 10.3$ & $233.8 \pm 39.3$ & $1.23 \pm 0.12$ & $0.59 \pm 0.06$ & $4.56 \pm 2.02$ & $0.75 \pm 0.16$ \\
\hline FCC 63 & $231.8 \pm 6.9$ & $167.3 \pm 32.2$ & $81.0 \pm 10.0$ & $2.34 \pm 0.06$ & $1.15 \pm 0.03$ & $8.66 \pm 3.56$ & $0.32 \pm 0.05$ \\
\hline FCC 193 & $38.6 \pm 7.1$ & $21.4 \pm 5.4$ & $12.6 \pm 4.8$ & $0.30 \pm 0.05$ & $0.16 \pm 0.03$ & $1.27 \pm 0.57$ & $0.37 \pm 0.16$ \\
\hline FCC 170 & $60.0 \pm 8.9$ & $52.6 \pm 8.2$ & $7.8 \pm 3.8$ & $0.63 \pm 0.09$ & $0.33 \pm 0.04$ & $3.04 \pm 1.70$ & $0.12 \pm 0.06$ \\
\hline FCC 153 & $48.1 \pm 8.0$ & $45.2 \pm 7.6$ & $3.9 \pm 2.5$ & $1.13 \pm 0.18$ & $0.60 \pm 0.10$ & $5.53 \pm 2.87$ & $0.07 \pm 0.05$ \\
\hline FCC 177 & $66.6 \pm 9.2$ & $61.7 \pm 8.8$ & $4.8 \pm 2.8$ & $1.63 \pm 0.22$ & $0.87 \pm 0.12$ & $9.98 \pm 6.30$ & $0.07 \pm 0.04$ \\
\hline FCC 47 & $286.6 \pm 13.4$ & $208.2 \pm 16.6$ & $78.5 \pm 9.8$ & $4.61 \pm 0.21$ & $2.51 \pm 0.11$ & $30.71 \pm 20.74$ & $0.27 \pm 0.03$ \\
\hline FCC 43 & $31.3 \pm 6.6$ & $27.2 \pm 6.0$ & $4.4 \pm 3.0$ & $0.83 \pm 0.17$ & $0.49 \pm 0.10$ & $10.46 \pm 12.93$ & $0.14 \pm 0.10$ \\
\hline FCC 190 & $141.2 \pm 13.2$ & $121.6 \pm 12.1$ & $14.1 \pm 5.3$ & $3.79 \pm 0.35$ & $2.00 \pm 0.18$ & $18.94 \pm 10.30$ & $0.10 \pm 0.04$ \\
\hline FCC 310 & $28.6 \pm 6.7$ & $25.4 \pm 5.9$ & $3.4 \pm 3.3$ & $0.65 \pm 0.15$ & $0.35 \pm 0.08$ & $3.20 \pm 1.82$ & $0.11 \pm 0.11$ \\
\hline FCC 249 & $146.2 \pm 13.4$ & $123.0 \pm 12.3$ & $22.9 \pm 5.6$ & $3.23 \pm 0.29$ & $1.79 \pm 0.16$ & $14.87 \pm 7.11$ & $0.15 \pm 0.04$ \\
\hline FCC 148 & $22.6 \pm 10.8$ & $22.5 \pm 8.2$ & $0.2 \pm 13.6$ & $0.41 \pm 0.19$ & $0.24 \pm 0.11$ & $2.10 \pm 1.42$ & $0.00 \pm 0.59$ \\
\hline FCC 255 & $68.8 \pm 9.2$ & $55.5 \pm 8.3$ & $12.9 \pm 4.3$ & $2.25 \pm 0.30$ & $1.28 \pm 0.17$ & $19.13 \pm 17.27$ & $0.18 \pm 0.06$ \\
\hline FCC 277 & $36.9 \pm 6.9$ & $28.3 \pm 6.0$ & $9.7 \pm 3.4$ & $1.08 \pm 0.20$ & $0.59 \pm 0.11$ & $4.88 \pm 2.38$ & $0.25 \pm 0.10$ \\
\hline FCC 55 & $29.2 \pm 6.1$ & $21.6 \pm 5.2$ & $7.9 \pm 3.2$ & $1.34 \pm 0.28$ & $0.74 \pm 0.15$ & $7.30 \pm 4.33$ & $0.26 \pm 0.12$ \\
\hline FCC 152 & $9.3 \pm 3.6$ & $8.5 \pm 3.4$ & $1.2 \pm 1.2$ & $0.39 \pm 0.15$ & $0.24 \pm 0.09$ & $2.66 \pm 1.91$ & $0.12 \pm 0.13$ \\
\hline FCC 301 & $15.0 \pm 5.3$ & $13.8 \pm 4.7$ & $1.9 \pm 2.8$ & $0.61 \pm 0.21$ & $0.34 \pm 0.12$ & $3.33 \pm 2.10$ & $0.11 \pm 0.18$ \\
\hline FCC 335 & $9.9 \pm 3.6$ & $9.9 \pm 3.6$ & $0.0 \pm 0.0$ & $0.74 \pm 0.26$ & $0.45 \pm 0.16$ & $7.82 \pm 7.79$ & $0.00 \pm 0.00$ \\
\hline NGC 134 & $347.5 \pm 20.8$ & $288.1 \pm 22.2$ & $59.4 \pm 7.6$ & $0.79 \pm 0.04$ & $0.44 \pm 0.02$ & $3.55 \pm 1.64$ & $0.17 \pm 0.02$ \\
\hline FCC 143 & $49.7 \pm 8.1$ & $44.7 \pm 7.6$ & $5.3 \pm 2.7$ & $1.96 \pm 0.32$ & $1.06 \pm 0.17$ & $9.71 \pm 5.34$ & $0.10 \pm 0.05$ \\
\hline FCC 95 & $9.8 \pm 3.9$ & $9.7 \pm 3.6$ & $-0.1 \pm 1.7$ & $0.68 \pm 0.26$ & $0.38 \pm 0.15$ & $3.57 \pm 2.29$ & $-0.01 \pm 0.17$ \\
\hline FCC 136 & $16.4 \pm 4.6$ & $15.6 \pm 4.5$ & $1.1 \pm 1.2$ & $1.54 \pm 0.43$ & $0.89 \pm 0.25$ & $8.32 \pm 4.89$ & $0.06 \pm 0.07$ \\
\hline FCC 182 & $37.5 \pm 8.3$ & $38.7 \pm 7.6$ & $1.5 \pm 3.0$ & $3.06 \pm 0.67$ & $1.67 \pm 0.36$ & $15.06 \pm 8.12$ & $0.03 \pm 0.07$ \\
\hline FCC 204 & $12.0 \pm 4.2$ & $11.6 \pm 4.0$ & $0.5 \pm 1.2$ & $1.14 \pm 0.39$ & $0.65 \pm 0.22$ & $6.63 \pm 4.53$ & $0.04 \pm 0.10$ \\
\hline FCC 119 & $11.6 \pm 4.6$ & $10.0 \pm 4.1$ & $1.7 \pm 2.2$ & $1.50 \pm 0.59$ & $0.88 \pm 0.34$ & $10.88 \pm 8.75$ & $0.14 \pm 0.19$ \\
\hline FCC 90 & $16.0 \pm 4.7$ & $12.7 \pm 4.2$ & $3.6 \pm 2.1$ & $1.68 \pm 0.49$ & $1.19 \pm 0.35$ & $13.29 \pm 8.98$ & $0.21 \pm 0.14$ \\
\hline FCC 26 & $14.8 \pm 4.3$ & $14.9 \pm 4.3$ & $0.0 \pm 0.0$ & $1.74 \pm 0.50$ & $1.42 \pm 0.41$ & $58.75 \pm 138.32$ & $0.00 \pm 0.00$ \\
\hline FCC 106 & $10.5 \pm 4.5$ & $8.8 \pm 4.0$ & $2.9 \pm 2.1$ & $1.17 \pm 0.49$ & $0.68 \pm 0.29$ & $7.25 \pm 5.57$ & $0.24 \pm 0.20$ \\
\hline FCC 19 & $7.6 \pm 3.6$ & $8.4 \pm 3.5$ & $0.0 \pm 0.0$ & $1.32 \pm 0.62$ & $0.82 \pm 0.38$ & $22.10 \pm 36.02$ & $0.00 \pm 0.00$ \\
\hline FCC 202 & $7.2 \pm 8.7$ & $4.7 \pm 6.0$ & $2.5 \pm 10.6$ & $1.02 \pm 1.23$ & $0.60 \pm 0.73$ & $6.66 \pm 9.00$ & $0.34 \pm 1.52$ \\
\hline FCC 324 & $12.9 \pm 4.4$ & $13.1 \pm 4.4$ & $0.0 \pm 0.0$ & $2.25 \pm 0.76$ & $1.32 \pm 0.45$ & $22.61 \pm 20.11$ & $0.00 \pm 0.00$ \\
\hline FCC 288 & $13.1 \pm 5.0$ & $11.1 \pm 4.4$ & $2.1 \pm 2.6$ & $2.22 \pm 0.84$ & $1.33 \pm 0.51$ & $19.83 \pm 17.92$ & $0.16 \pm 0.20$ \\
\hline FCC 303 & $12.8 \pm 4.2$ & $13.3 \pm 4.2$ & $0.0 \pm 0.0$ & $1.48 \pm 0.48$ & $0.89 \pm 0.29$ & $24.30 \pm 38.50$ & $0.00 \pm 0.00$ \\
\hline FCC 203 & $21.6 \pm 5.7$ & $19.9 \pm 5.3$ & $1.8 \pm 2.3$ & $3.50 \pm 0.92$ & $2.10 \pm 0.55$ & $23.22 \pm 15.13$ & $0.08 \pm 0.10$ \\
\hline FCC 100 & $23.6 \pm 5.7$ & $23.0 \pm 5.6$ & $0.2 \pm 1.3$ & $4.49 \pm 1.07$ & $2.87 \pm 0.69$ & $27.26 \pm 14.51$ & $0.01 \pm 0.05$ \\
\hline IC 2006 & $121.7 \pm 6.4$ & $55.0 \pm 8.9$ & $66.7 \pm 6.2$ & $1.21 \pm 0.06$ & $0.60 \pm 0.03$ & $5.04 \pm 2.43$ & $0.54 \pm 0.05$ \\
\hline
\end{tabular}

Note. Column (1): the ID of galaxies. Column (2): total number of GCs. Column (3): total number of blue GCs. Column (4): total number of red GCs. Column (5): specific frequency. Column (6): specific frequency in $z$ bandpass. Column (7): $N_{\mathrm{GC}}$ normalized to stellar mass of $10^{9} M_{\odot}$. Column (8): fraction of red GCs.

constrain the shape of its density profile using data at larger radii. Also observed as part of the ACSFCS, FCC 202 is only $4 ! 6$ away from FCC 213. The GC candidates in the FCC 202 ACS field are evenly distributed throughout the image without any evidence of central concentration, implying that these GCs mostly belong to the GC system of the nearby massive galaxy. Therefore, we assume that the GCs outside of $6 R_{e}$ for FCC 202 ( $\sim$ half the size of the FOV) belong solely to the halo of FCC 213. We divide them into five bins of distance from GCC 213, with equal GC numbers in each bin, and use them as complementary density measurements outside the ACS image of FCC 213. In the even outer regions, Dirsch et al. (2003) conducted a wide-field study of NGC 1399 and sampled its GC system as far as $23^{\prime}$. We take the measurements between $4^{\prime}$ and $23^{\prime}$ galactocentric radius from the Table 3 in their paper and include them in our profile fitting. With 10 annuli of equal GC numbers, we fit the Sérsic profile of its GC number density and integrate it to infinity to estimate the total number of FCC 213's GC system. Dirsch et al. (2003) found $6450 \pm 700 \mathrm{GCs}$ within a radius of $15^{\prime}(83 \mathrm{kpc})$, in agreement with ours in the same area.

FCC 21 (NGC 1316). Unlike most local, massive ETGs, FCC 21 has experienced a relatively recent major merger that happened about $3 \mathrm{Gyr}$ ago (Goudfrooij et al. 2001b). It contains a significant amount of intermediate-age GCs with relatively high metallicity, which possibly formed during the merger event (e.g., Goudfrooij et al. 2004). This subpopulation of GCs is relatively faint and red. Most ETGs in our sample have GCLFs with a similar mean and standard deviation (Villegas et al. 2010). However, the GCLF of FCC 21 is 
peaked at a significantly fainter magnitude with a much wider dispersion. Taking into account the brighter surface brightness of FCC 21, we only select out the GC candidates that are brighter than the mean of the GCLF to avoid problems with nondetection. Also, FCC 21 is in a special case in profile fitting. We find that the individual fitting of the blue and red GC populations (see Section 3.2 below) is better than the profile fitting of its entire GC system, and we adopt the total GC number to be the sum of these two populations. Furthermore, the closest companion of FCC 21 , FCC 19, is a low-mass ETG with a small GC system. No excess of GCs is detected in its outskirts, indicating that it is not contaminated by FCC 21 . We have checked the fitted GC number density profile of FCC 21 and find that it has fallen to zero at a radius smaller than the distance from FCC 19.

FCC 219, FCC 184, FCC 182, and FCC 202. The GC systems of these galaxies are contaminated by FCC 213 . We subtract the contaminants from FCC 213 using its GC number density profile. For FCC 202, we only take into account the GCs inside $6 R_{e}$, which corresponds to half the area of the FOV.

FCC 148. This is a low-mass ETG with a close massive companion, FCC 147. The projected distance between them is comparable to the size of the ACS FOV, and a quarter of its image is filled with the GCs from FCC 147. However, in the area opposite the contaminated region, few GCs are detected. Therefore, when counting its GCs, we used only the central area dominated by the GC system of FCC 148 itself. We then divided the outer region equally into two sections, leaving most of the contaminants from FCC 147 in one of them. The GC number in the central area is counted after the subtraction of contaminants that are estimated from the fitted density profile. In the outer region, we measure the GC numbers in the half of the image with few contaminants, then double it. The total number of GCs is the sum of the counts from both the inner and outer areas.

\subsection{Separation of Blue and Red GCs}

Because the dividing line of the blue and red GC populations does not vary much among ETGs (Peng et al. 2006), we simply separate these two populations at $g-z=1.16$, which is the same cut used in Peng et al. (2008). We calculate the number in each GC subpopulation in the same way as for counting the total numbers but only extrapolate the density profiles for the ETGs for those galaxies with $M_{z}<-21$ (as well as IC 2006) when counting red GCs. If the profile fitting of the blue population has large uncertainty (due to being more spatially extended), we estimate the number by subtracting the number of the better-constrained red population from the total number.

We note that the merger remnant FCC 21 contains a substantial fraction of intermediate-age GCs, especially in the outer regions. This means that the color distribution of its GC system has a significant red peak. There is still a bimodal color distribution, however (Goudfrooij et al. 2001a, 2004), and the color cut should probably be different from the one we use. Goudfrooij et al. (2004) suggested the blue and red peaks at $B-I=1.5$ and 1.8 and a possible separation at 1.65 . However, because we do not want to add additional layers of interpretation with a movable color cut, we decided to apply a homogeneous color cut on all galaxies and consider our measurements of blue and red GCs in FCC 21 as simply a reference for comparing with the rest of the sample, keeping in mind the special nature of this galaxy.
Another special case is FCC 213. Part of the data used for its profile fitting are from Dirsch et al. (2003). In their study, both the photometry systems and the color separation were different from ours. However, to constrain the profile shapes, we adopt the number densities of blue and red GCs at radii between $16^{\prime}$ and $23^{\prime}$ in Dirsch et al. (2003). Accordingly, the results of the blue or red GCs of the galaxies that are contaminated by its GC systems are not on exactly the same scale as others. Fortunately, except for FCC 202, the contaminants from FCC 213 account for no more than $15 \%$ of their GC numbers. Furthermore, the systematic errors caused by color calibrations should not affect our results qualitatively.

\section{Results}

Traditionally, $S_{N}$ is defined as the number of GCs per unit galactic stellar $V$-band luminosity (Harris \& van den Bergh 1981). Peng et al. (2008) introduced the modified parameter, $S_{N, z}$ (Equation (1)), that is similar to $S_{N}$ but normalized to the galaxy luminosity of $M_{z}=-15$, with the $z$-band being a redder bandpass that more accurately reflects the stellar mass. Similarly, Zepf \& Ashman (1993) defined $T$ as the number of GCs per $10^{9} M_{\odot}$ of galactic stellar mass (Equation (2)), which has the advantage of comparing galaxies with different stellar mass-to-light ratios $\left(M_{\star} / L\right)$ :

$$
\begin{aligned}
& S_{N, z}=N_{\mathrm{GC}} \times 10^{0.4\left(M_{z}+15\right)}, \\
& T=N_{\mathrm{GC}} /\left(M_{G^{\star}} / 10^{9} M_{\odot}\right) .
\end{aligned}
$$

All of these parameters indicate the GC formation (and survival) efficiency relative to that of field stars, and they are listed in Table 3. Because $z$-band magnitude is a better indicator of stellar mass than $V$-band, and we have actually measured $M_{z}$ for our sample galaxies, $S_{N, z}$ is better than $S_{N}$ for our purposes. Furthermore, our samples are all ETGs, which have similar $M_{\star} / L$. We have tested that in all the analysis in this work, $S_{N, z}$ and $T$ have similar trends in relation to other parameters. Because the measurement of stellar mass relies on stellar population models that would bring in additional systematic uncertainties, we primarily use $S_{N, z}$ in our analysis.

\subsection{Dependence on Galactic Mass}

Figure 1 displays the relation between $S_{N, z}$ and $M_{z}$ of the 143 ETGs in the ACSFCS and ACSVCS. The measurements for Virgo galaxies are adopted directly from Peng et al. (2008). Red circles and blue dots represent the ETGs from Fornax and Virgo, respectively. From top to bottom, the $y$-axis is $S_{N, z}$ measured for the entire GC system, the blue GC population, and the red GC population, respectively.

The distributions of all three parameters are similar in Fornax and Virgo. Specifically, the $S_{N, z}$ for all GCs is roughly constant with some scatter but largely below $S_{N, z}=1.5$ among intermediate-mass ETGs, and it increases with galactic luminosity at the bright end $\left(M_{z}<-23\right)$. It also systematically rises at the faint end, but with large scatter. While the lowest values reach zero, the highest values are comparable or even higher than the highest $S_{N, z}$ at the massive end in their host clusters.

However, the $\mathrm{U}$ shape of this relation is mainly driven by the ETGs in Virgo. This may simply be a result of the smaller sample size in Fornax. At the bright end, only two ETGs from Fornax have $M_{z}$ brighter than -23 . While the BCG FCC 213 has a high $S_{N, z}$ that follows the U-shaped distribution in Virgo, 


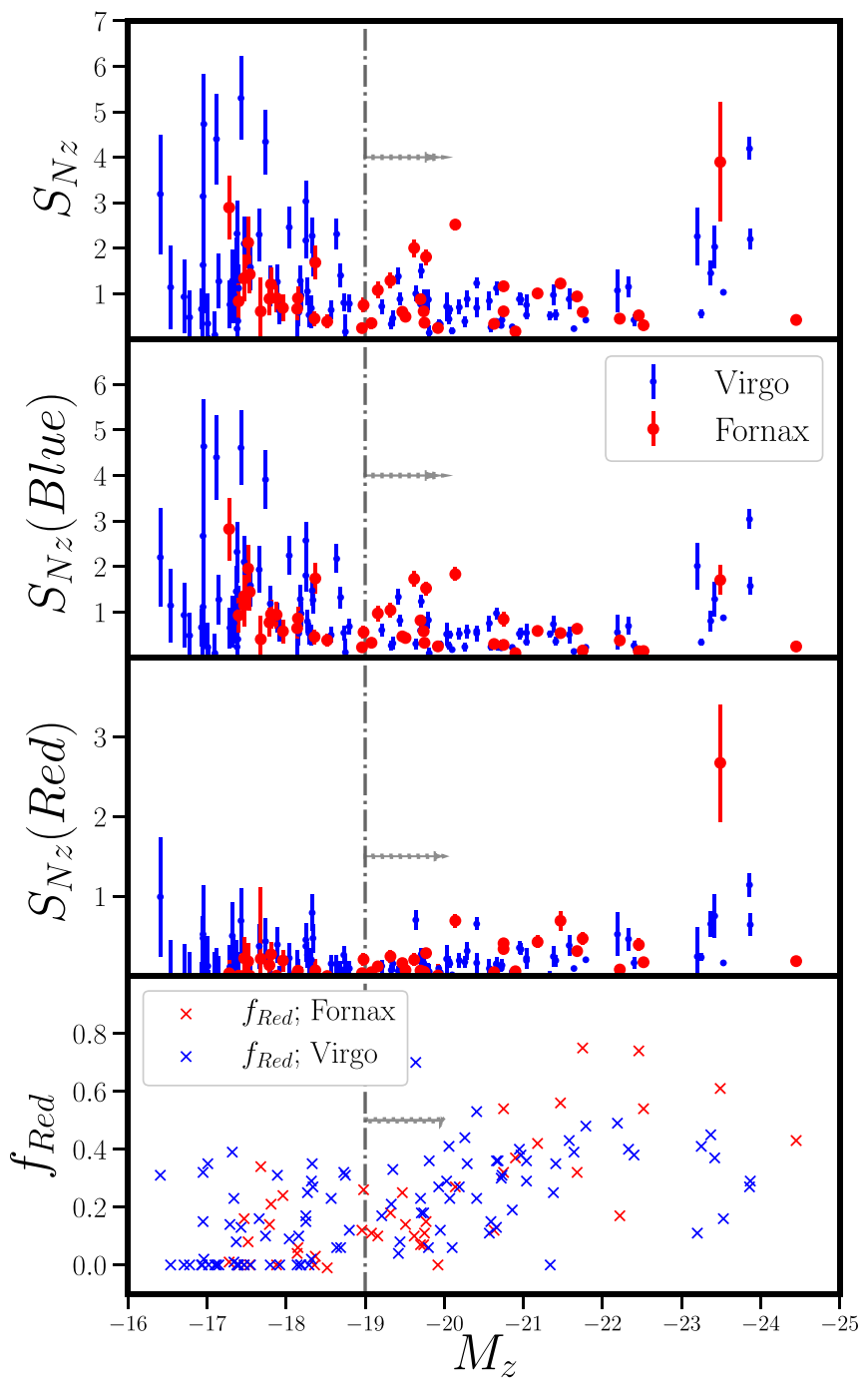

Figure 1. Relation between $S_{N, z}$ and $z$-band absolute magnitudes of the 143 ETGs from ACSFCS (red circles) and ACSVCS (blue dots) samples. The $S_{N, z}$ values in the first three panels are measured by the total GC population, blue GCs, and red GCs in galaxies, respectively. The $S_{N, z}$ of the entire GC and blue GC populations have similar trends with $M_{z}$. They have a roughly constant value for intermediatemass ETGs and increase with galactic luminosity at the bright end $\left(M_{z}<-23\right)$. They also systematically rise at the faint end, but with large scatter. The vertical dotted-dashed lines show the dividing line for low-mass ETGs defined in Peng et al. (2008), but we redefine it as $M_{z}<-20$ (illustrated by the arrows) in this work. The $S_{N, z}$ values of red GCs are nearly all below $\sim 1$, slightly increasing with $M_{z}$ among intermediate-mass ETGs. In the bottom panel, the red and blue crosses illustrate the relation between $f_{\text {Red }}$ ( $y$-axis on the right) and $M_{z}$ in the Fornax and Virgo Clusters. The low-mass ETGs fainter than $M_{z}=-20$ have relatively low $f_{\text {Red }}$, below 0.4 , and some of the faintest ETGs have no red GC. Here $f_{\text {Red }}$ is positively correlated with $M_{z}$ in the intermediate-mass range and flattens or decreases with $M_{z}$ at the high-mass end.

the other one, FCC 21, is a post-starburst galaxy and has a low $S_{N, z}$ close to zero. This low $S_{N, z}$ is possibly due to the high luminosity produced by its relatively young stellar population.

At the faint end, because Fornax is $3.5 \mathrm{Mpc}$ farther than Virgo, the ACSFCS did not sample the ETGs fainter than $M_{z} \sim-17$. In the range of magnitudes that overlap, the ETGs with the highest $S_{N, z}$ at each magnitude are mostly from Virgo. In Virgo, there are galaxies with $S_{N, z}$ higher than that for the BCG (M87), while this is not the case in Fornax.

Peng et al. (2008) drew the dividing line between low- and intermediate-mass galaxies at $M_{z}=-19$ mag (vertical dotteddashed lines in Figure 1) when only using the ETGs from
Virgo. However, the scatter starts to increase upward at $M_{z}=-20 \mathrm{mag}$ after including the samples from Fornax. It is driven by two ETGs, FCC 190 and FCC 249. Because they are not special in morphology, color, and location, we define $M_{z}=-20 \mathrm{mag}$ as the dividing line in this work, which is shown by the arrows. One possible reason why Peng et al. (2008) did not find an increase of scatter at the brighter limit of $M_{z} \gtrsim-20 \mathrm{mag}$ is that the ACSVCS is incomplete for $B_{T}>12.15 \mathrm{mag}\left(M_{B}>-18.94 \mathrm{mag}\right)$ and misses 63 ETGs from the parent sample. The typical $B-z$ color of massive ETGs is $\sim 2 \mathrm{mag}$, and the dividing line we choose in this work corresponds to $M_{B} \gtrsim-18 \mathrm{mag}$, which is a traditional cut for defining early-type dwarf galaxies in observations.

An outlier from this relation is FCC 47, which has $S_{N, z}=2.51 \pm 0.11$ at $M_{z}=-20.14 \mathrm{mag}$. It has the appearance of a normal ETG, and its color lies on the conventional ETG color-magnitude relation. However, it has the largest 3D clustercentric distance in the ACSFCS sample (Blakeslee et al. 2009), at $\sim 2$ times the virial radius of the Fornax Cluster. From the projected galaxy distribution in Fornax, FCC 47 is relatively isolated. One hypothesis is that it is an infalling central galaxy with a higher total mass-to-light ratio, resembling the behavior of the most massive ETGs. Its GC system has possibly experienced fewer external disruption processes, and the GCs may have a higher survival efficiency.

The blue GCs are the dominant populations in most ETGs. The distribution of $S_{N, z}$ is significantly biased by them. In the middle panel, the $S_{N, z}$ of blue GCs mimics the distribution of $S_{N, z}$ in the top panel. Low-mass ETGs have few to zero red GCs, something that is likely due to their low stellar metallicities. At higher masses, $S_{N, z \text {,red }}$ slightly increases with $M_{z}$, on average, among both the intermediate- and highestmass ETGs.

A more direct way to study the formation ability of red GCs is to look at their fraction of the total GC population. In the bottom panel, the red and blue crosses illustrate the relationship between $f_{\text {Red }}$ and $M_{z}$ in the Fornax and Virgo Clusters. The low-mass ETGs that are fainter than $M_{z}=-20$ mag have relatively low $f_{\text {Red }}$ scattered below 0.4 . A fraction of the faintest ETGs do not have any red GCs. As with just the Virgo data, we find that $f_{\text {Red }}$ is positively correlated with $M_{z}$ in the intermediate-mass range and either flattens or decreases with $M_{z}$ at the high-mass end. Interestingly, among the massive ETGs, the galaxies with the highest $f_{\text {Red }}$ are all in Fornax. In addition, the shape of the $f_{\text {Red }}$ distribution shows that the rise in $f_{\text {Red }}$ starts at $M_{z}=-20 \mathrm{mag}$.

If all GCs formed in situ, then $S_{N, z}$ would be a simple expression of the GC formation efficiency in that galaxy. However, because the outer halos of massive ETGs are built by the accretion of low-mass satellites, the measured $S_{N, z}$ is actually a combination of the GC formation efficiencies from all of the current galaxy's progenitors. If we want to measure the efficiency of in situ formation of the most massive progenitor, these GCs are likely to be at the galaxy's center. Typically, it is assumed that the in situ GC components are the red GCs, and the behavior of $S_{N, z \text {,red }}$ is described above, but we can also avoid imposing this interpretation and instead look only at the behavior of the innermost GCs. We therefore also measure $S_{N, z}$ within the innermost $1 R_{e}$ to study the in situ GC formation of massive galaxies (Table 4). For the massive galaxies in both ACSFCS and ACSVCS, we isolate their central $1 R_{e}$ area, count the GCs therein, and use the $z$-band 


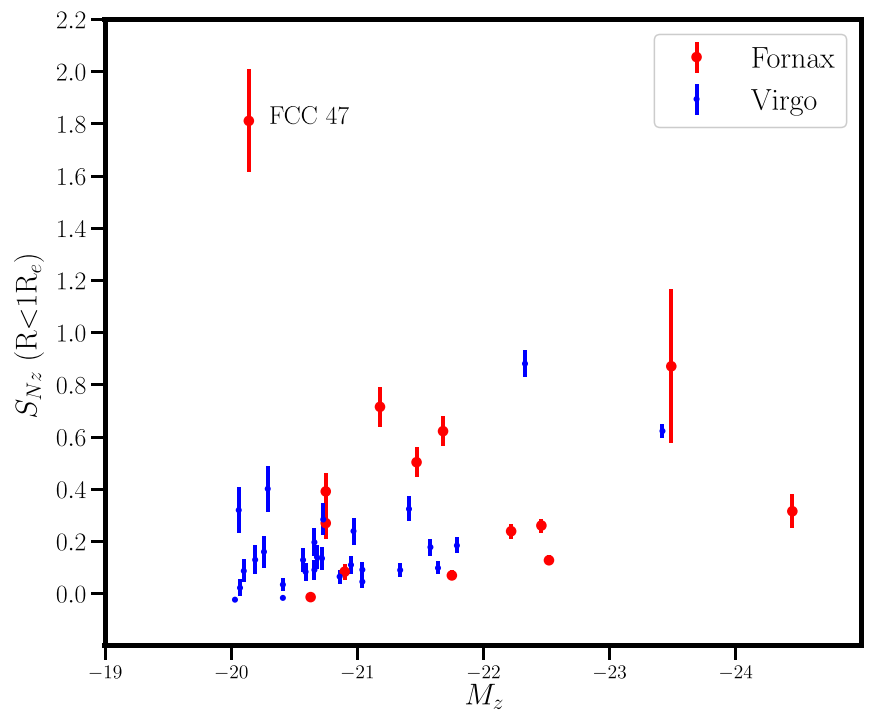

Figure 2. The $S_{N, z}$ measured for the inner $1 R_{e}$ of massive ETGs in the ACSFCS (red circles) and ACSVCS (blue dots) samples and their relation to galactic $z$ band absolute magnitude. Excluding FCC 47, the outlier with a significantly high value, the brighter galaxies have higher inner $S_{N, z}$.

luminosity of this area (i.e., half the total luminosity). The most massive ACSVCS galaxies have $R_{e}$ larger than the FOV, and we do not include them.

Figure 2 shows the relation between this inner (in situ) $S_{N, z}$ and luminosities of massive galaxies. Excluding FCC 47, the outlier with a significantly high value, the brighter galaxies generally have higher inner $S_{N, z}$.

Regarding the outlier FCC 47, it has several special properties. It has the largest $3 \mathrm{D}$ clustercentric distance of the ACSFCS sample. It is located at $\sim 2$ times the viral radius of the Fornax Cluster. Although it is relatively faint among massive galaxies, it has a rich GC system, which makes its $S_{N, z}$ higher than others with similar masses. Furthermore, according to Liu et al. (2016c), it has a significant sample of diffuse star clusters. However, we are not able to draw any conclusions for it with the limited information.

\subsection{Dependence on Environment}

Peng et al. (2008) found that the low-mass ETGs at smaller clustercentric radii in Virgo have higher average $S_{N}$, indicating that the low-mass ETGs in the denser environments have higher GC formation efficiencies. In addition, they suggested that the environmental density is the second parameter that drives the large scatter of $S_{N, z}$ at low masses. Correspondingly, simulation work by Mistani et al. (2016) found that dwarf galaxies in denser environments quenched earlier, boosting their total mass-to-light ratios and $S_{N}$. In this section, we further explore the environmental effects on the GC formation in lowmass ETGs from the two clusters.

In order to compare the environments in different clusters, we bring in two parameters to estimate the environment, following Guérou et al. (2015). One is $\Sigma_{15}$, defined as the number of galaxies per square degree within a region that includes the 15 closest neighbors, indicating the number density of the local environment. The other one is $L_{\max } / L$, the luminosity ratio between the brightest galaxy among the 15 closest neighbors and the galaxy itself. It generally reflects the mass ratio between the hosts and their neighbor galaxies. In the
Table 4

$S_{N, z}$ within $1 R_{e}$ of Massive Galaxies in ACSFCS and ACSVCS

\begin{tabular}{|c|c|c|c|c|c|}
\hline $\begin{array}{l}\text { ID } \\
(1)\end{array}$ & $\begin{array}{c}R_{e, \mathrm{gal}} \\
(\operatorname{arcsec}) \\
(2)\end{array}$ & $\begin{array}{c}n_{\text {gal }} \\
(3)\end{array}$ & $\begin{array}{c}M_{z, \text { In }} \\
(4)\end{array}$ & $\begin{array}{c}N_{\mathrm{GC}, \mathrm{In}} \\
\quad(5)\end{array}$ & $\begin{array}{l}S_{N, z, \text { In }} \\
\text { (6) }\end{array}$ \\
\hline F21 & 146.03 & 6.29 & -23.69 & $950.76 \pm 192.33$ & $0.31 \pm 0.06$ \\
\hline F213 & 114.58 & 7.51 & -22.73 & $1083.65 \pm 364.82$ & $0.87 \pm 0.29$ \\
\hline F219 & 22.91 & 3.85 & -21.76 & $65.11 \pm 8.91$ & $0.12 \pm 0.01$ \\
\hline F167 & 30.33 & 4.0 & -21.70 & $125.77 \pm 12.34$ & $0.26 \pm 0.02$ \\
\hline F276 & 35.70 & 5.39 & -20.92 & $146.23 \pm 13.36$ & $0.62 \pm 0.05$ \\
\hline F147 & 29.20 & 5.13 & -20.71 & $97.48 \pm 10.89$ & $0.50 \pm 0.05$ \\
\hline F83 & 26.56 & 4.98 & -20.42 & $106.09 \pm 11.34$ & $0.71 \pm 0.07$ \\
\hline F184 & 21.37 & 4.14 & -20.99 & $17.47 \pm 4.79$ & $0.06 \pm 0.01$ \\
\hline F63 & 14.67 & 3.0 & -19.99 & $39.06 \pm 6.92$ & $0.39 \pm 0.06$ \\
\hline F193 & 11.97 & 2.72 & -20.14 & $9.63 \pm 3.38$ & $0.08 \pm 0.02$ \\
\hline $\mathrm{F} 170$ & 6.83 & 2.0 & -19.87 & $-1.19 \pm 0.0$ & $-0.01 \pm 0.0$ \\
\hline $\mathrm{F} 47$ & 27.65 & 3.08 & -19.38 & $103.04 \pm 11.19$ & $1.81 \pm 0.19$ \\
\hline N1340 & 33.21 & 3.78 & -21.46 & $92.37 \pm 10.71$ & $0.23 \pm 0.02$ \\
\hline I2006 & 18.94 & 3.50 & -19.99 & $26.94 \pm 5.87$ & $0.27 \pm 0.05$ \\
\hline V1978 & 98.48 & 4.85 & -22.66 & $726.75 \pm 29.93$ & $0.62 \pm 0.02$ \\
\hline V1632 & 84.37 & 7.60 & -21.57 & $376.59 \pm 21.76$ & $0.88 \pm 0.05$ \\
\hline V1231 & 18.64 & 3.22 & -20.82 & $38.13 \pm 6.91$ & $0.17 \pm 0.03$ \\
\hline V2095 & 14.29 & 3.96 & -20.19 & $13.20 \pm 3.96$ & $0.11 \pm 0.03$ \\
\hline V1154 & 29.50 & 5.07 & -21.03 & $47.95 \pm 7.74$ & $0.18 \pm 0.02$ \\
\hline V1062 & 14.21 & 3.14 & -20.58 & $15.49 \pm 4.29$ & $0.09 \pm 0.02$ \\
\hline V2092 & 31.45 & 5.09 & -20.88 & $22.27 \pm 5.35$ & $0.09 \pm 0.02$ \\
\hline V369 & 7.74 & 2.76 & -19.65 & $2.51 \pm 1.73$ & $0.03 \pm 0.02$ \\
\hline V759 & 27.27 & 3.53 & -20.65 & $59.43 \pm 8.54$ & $0.32 \pm 0.04$ \\
\hline V1692 & 9.34 & 2.73 & -20.28 & $5.95 \pm 2.91$ & $0.04 \pm 0.02$ \\
\hline V2000 & 9.44 & 4.45 & -19.90 & $18.06 \pm 4.78$ & $0.19 \pm 0.05$ \\
\hline V685 & 11.20 & 3.21 & -20.28 & $11.92 \pm 3.95$ & $0.09 \pm 0.03$ \\
\hline V1664 & 15.91 & 4.33 & -20.21 & $29.26 \pm 6.10$ & $0.23 \pm 0.05$ \\
\hline V654 & 20.78 & 4.03 & -19.83 & $7.20 \pm 2.92$ & $0.08 \pm 0.03$ \\
\hline V944 & 11.61 & 3.35 & -19.90 & $8.35 \pm 3.37$ & $0.09 \pm 0.03$ \\
\hline V1938 & 13.36 & 4.11 & -20.10 & $7.20 \pm 2.92$ & $0.06 \pm 0.02$ \\
\hline V1279 & 12.01 & 2.15 & -19.96 & $13.10 \pm 4.12$ & $0.13 \pm 0.04$ \\
\hline V1720 & 27.22 & 4.46 & -19.92 & $13.10 \pm 4.12$ & $0.14 \pm 0.04$ \\
\hline V355 & 8.83 & 3.69 & -19.65 & $-1.19 \pm 0.0$ & $-0.01 \pm 0.0$ \\
\hline V1619 & 9.98 & 1.66 & -19.43 & $7.72 \pm 3.25$ & $0.12 \pm 0.05$ \\
\hline V1883 & 25.02 & 4.57 & -19.97 & $27.82 \pm 5.87$ & $0.28 \pm 0.05$ \\
\hline V1242 & 16.67 & 3.36 & -19.53 & $26.23 \pm 5.71$ & $0.40 \pm 0.08$ \\
\hline V784 & 13.67 & 3.00 & -19.50 & $10.18 \pm 3.82$ & $0.16 \pm 0.06$ \\
\hline V778 & 5.19 & 3.03 & -19.27 & $-1.19 \pm 0.0$ & $-0.02 \pm 0.0$ \\
\hline V828 & 10.50 & 2.20 & -19.31 & $1.19 \pm 1.68$ & $0.02 \pm 0.03$ \\
\hline V1250 & 11.78 & 7.97 & -19.34 & $4.76 \pm 2.38$ & $0.08 \pm 0.04$ \\
\hline V1630 & 13.11 & 2.24 & -19.30 & $16.90 \pm 4.64$ & $0.32 \pm 0.08$ \\
\hline V1025 & 10.12 & 3.71 & -19.81 & $10.90 \pm 3.79$ & $0.12 \pm 0.04$ \\
\hline
\end{tabular}

Note. Column (1): ID of massive galaxies. Columns (2) and (3): galactic $R_{e}$ in units of arcseconds and Sérsic index, adopted from measurements used in Turner et al. (2012) and Ferrarese et al. (2006) for ACSFCS and ACSVCS galaxies, respectively. Column (4): $z$-band absolute magnitude within $1 R_{e}$ of galactic main bodies. Columns (5) and (6): total number of GCs and $S_{N, z}$ within $1 R_{e}$ of the stars, respectively.

Virgo Cluster, our calculation is based on the complete parent sample of 163 ETGs. Our $L_{\max } / L$ is calculated in the $B$ band. Tables 5 and 6 list the results of both parameters of the ACSFCS and ACSVCS galaxies, respectively.

Note that we have also tried using $\Sigma_{5}$ and $L_{\max } / L$, calculated with the five closest neighbors, and the results are generally the same. Both $\Sigma_{15}$ and $L_{\max } / L$ are calculated using projected distances. Although the line-of-sight distances of ACSFCS and ACSVCS galaxies are provided by Blakeslee et al. (2009), we decided not to use them in our analysis 
Table 5

Environmental Parameters of ACSFCS Galaxies

\begin{tabular}{|c|c|c|}
\hline $\begin{array}{l}\text { ID } \\
(1)\end{array}$ & $\underset{(2)}{L_{\max } / L}$ & $\begin{array}{c}\Sigma_{15}\left(L_{\odot} \mathrm{pc}^{-2}\right) \\
(3)\end{array}$ \\
\hline$\overline{\text { FCC } 21}$ & 0.093 & 0.032 \\
\hline FCC 213 & 0.735 & 0.605 \\
\hline FCC 219 & 1.359 & 0.448 \\
\hline FCC 167 & 1.793 & 0.468 \\
\hline FCC 276 & 2.710 & 0.243 \\
\hline FCC 147 & 3.262 & 0.419 \\
\hline FCC 83 & 2.520 & 0.072 \\
\hline FCC 184 & 5.107 & 0.507 \\
\hline FCC 63 & 3.747 & 0.020 \\
\hline FCC 193 & 7.341 & 0.499 \\
\hline FCC 170 & 8.227 & 0.424 \\
\hline FCC 153 & 4.967 & 0.109 \\
\hline FCC 177 & 10.531 & 0.313 \\
\hline FCC 47 & 3.651 & 0.090 \\
\hline FCC 43 & 6.614 & 0.039 \\
\hline FCC 190 & 14.225 & 0.480 \\
\hline FCC 310 & 13.993 & 0.077 \\
\hline FCC 249 & 14.676 & 0.043 \\
\hline FCC 148 & 16.296 & 0.547 \\
\hline FCC 255 & 15.274 & 0.078 \\
\hline FCC 277 & 16.616 & 0.163 \\
\hline FCC 55 & 6.396 & 0.022 \\
\hline FCC 152 & 14.456 & 0.027 \\
\hline FCC 301 & 26.291 & 0.484 \\
\hline FCC 335 & 24.470 & 0.272 \\
\hline NGC 1340 & 0.985 & 0.009 \\
\hline FCC 143 & 26.400 & 0.434 \\
\hline FCC 95 & 19.269 & 0.142 \\
\hline FCC 136 & 53.977 & 0.416 \\
\hline FCC 182 & 48.650 & 0.400 \\
\hline FCC 204 & 23.570 & 0.034 \\
\hline FCC 119 & 31.005 & 0.053 \\
\hline FCC 90 & 58.541 & 0.243 \\
\hline FCC 26 & 16.616 & 0.073 \\
\hline FCC 106 & 30.878 & 0.080 \\
\hline FCC 19 & 226.324 & 0.026 \\
\hline FCC 202 & 66.951 & 0.642 \\
\hline FCC 324 & 76.500 & 0.133 \\
\hline FCC 288 & 75.278 & 0.073 \\
\hline FCC 303 & 67.181 & 0.054 \\
\hline FCC 203 & 77.878 & 0.209 \\
\hline FCC 100 & 46.994 & 0.117 \\
\hline IC 2006 & 4.863 & 0.285 \\
\hline
\end{tabular}

Note. Both $L_{\max } / L$ and $\Sigma_{15}$ are calculated in the $B$ band.

because the relatively large error bars of the 3D distances would smear out the relations shown in the plots. The line-ofsight depth of the Fornax cluster is not resolved by the surface brightness distances used in Blakeslee et al. (2009), so including these distances is of limited value.

Figure 3 displays the relations between $S_{N, z}$ and $\Sigma_{15}$. The relations for massive and low-mass ETGs are shown in the upper and lower panels, respectively. In the upper panel, the measurements are limited to the inner $1 R_{e}$, the same as in Figure 2. There is no clear environmental dependence in this plot, implying that the in situ formation of GCs in massive ETGs does not depend on the present-day environment (although environmental factors at higher redshift may have played a role). In the lower panel, from a global view, the
Table 6

Environmental Parameters of ACSVCS Galaxies

\begin{tabular}{|c|c|c|}
\hline $\begin{array}{l}\text { ID } \\
\text { (1) }\end{array}$ & $\underset{(2)}{L_{\max } / L}$ & $\begin{array}{c}\Sigma_{15}\left(L_{\odot} \mathrm{pc}^{-2}\right) \\
(3)\end{array}$ \\
\hline VCC 1226 & 0.304 & 1.826 \\
\hline VCC 1316 & 0.091 & 0.589 \\
\hline VCC 1978 & 0.310 & 0.404 \\
\hline VCC 881 & 1.0 & 0.511 \\
\hline VCC 798 & 0.161 & 0.044 \\
\hline VCC 763 & 1.0 & 0.404 \\
\hline VCC 731 & 1.629 & 0.453 \\
\hline VCC 1535 & 3.280 & 2.116 \\
\hline VCC 1903 & 3.221 & 0.422 \\
\hline VCC 1632 & 0.319 & 0.113 \\
\hline VCC 1231 & 3.162 & 0.540 \\
\hline VCC 2095 & 3.564 & 0.500 \\
\hline VCC 1154 & 1.158 & 0.322 \\
\hline VCC 1062 & 0.586 & 0.053 \\
\hline VCC 2092 & 5.495 & 0.344 \\
\hline VCC 369 & 5.597 & 0.132 \\
\hline VCC 759 & 0.258 & 0.082 \\
\hline VCC 1692 & 10.185 & 0.198 \\
\hline VCC 1030 & 5.199 & 0.820 \\
\hline VCC 2000 & 9.462 & 1.951 \\
\hline VCC 685 & 6.194 & 0.147 \\
\hline VCC 1664 & 2.831 & 0.314 \\
\hline VCC 654 & 6.426 & 0.152 \\
\hline VCC 944 & 1.706 & 0.050 \\
\hline VCC 1938 & 8.165 & 0.485 \\
\hline VCC 1279 & 10.964 & 0.735 \\
\hline VCC 1720 & 0.398 & 0.019 \\
\hline VCC 355 & 1.202 & 0.031 \\
\hline VCC 1619 & 5.105 & 0.151 \\
\hline VCC 1883 & 21.478 & 0.133 \\
\hline VCC 1242 & 3.837 & 0.375 \\
\hline VCC 784 & 2.208 & 0.053 \\
\hline VCC 1537 & 1.870 & 0.206 \\
\hline VCC 778 & 2.831 & 0.103 \\
\hline VCC 1321 & 2.728 & 0.065 \\
\hline VCC 828 & 11.376 & 0.496 \\
\hline VCC 1250 & 20.511 & 0.795 \\
\hline VCC 1630 & 6.854 & 0.136 \\
\hline VCC 1146 & 14.859 & 0.544 \\
\hline VCC 1025 & 18.535 & 0.986 \\
\hline VCC 1303 & 34.355 & 0.274 \\
\hline VCC 1913 & 35.645 & 0.829 \\
\hline VCC 1327 & 26.302 & 0.554 \\
\hline VCC 1125 & 3.872 & 0.252 \\
\hline VCC 1475 & 3.801 & 0.033 \\
\hline VCC 1178 & 49.203 & 0.749 \\
\hline VCC 1283 & 21.281 & 0.520 \\
\hline VCC 1261 & 1.690 & 0.132 \\
\hline VCC 698 & 4.325 & 0.257 \\
\hline VCC 1422 & 7.798 & 0.073 \\
\hline VCC 2048 & 40.179 & 0.160 \\
\hline VCC 1871 & 51.999 & 0.710 \\
\hline VCC 9 & 10.764 & 0.191 \\
\hline VCC 575 & 51.522 & 0.809 \\
\hline VCC 1910 & 64.268 & 0.309 \\
\hline VCC 1049 & 103.752 & 1.193 \\
\hline VCC 856 & 11.376 & 0.056 \\
\hline VCC 140 & 14.321 & 0.082 \\
\hline VCC 1355 & 13.551 & 0.291 \\
\hline VCC 1087 & 10.471 & 0.262 \\
\hline VCC 1297 & 88.715 & 0.570 \\
\hline VCC 1861 & 76.559 & 1.852 \\
\hline VCC 543 & 16.904 & 0.073 \\
\hline
\end{tabular}


Table 6

(Continued)

\begin{tabular}{|c|c|c|}
\hline $\begin{array}{l}\text { ID } \\
(1)\end{array}$ & $L_{\text {max }} / L$ & $\begin{array}{c}\Sigma_{15}\left(L_{\odot} \mathrm{pc}^{-2}\right) \\
(3)\end{array}$ \\
\hline VCC 1431 & 9.549 & 0.275 \\
\hline VCC 1528 & 29.376 & 0.189 \\
\hline VCC 1695 & 29.107 & 0.096 \\
\hline VCC 1833 & 4.325 & 0.006 \\
\hline VCC 437 & 65.463 & 0.088 \\
\hline VCC 2019 & 2.355 & 0.022 \\
\hline VCC 33 & 27.542 & 0.166 \\
\hline VCC 200 & 60.813 & 0.061 \\
\hline VCC 571 & 77.268 & 2.080 \\
\hline VCC 21 & 14.723 & 0.013 \\
\hline VCC 1488 & 17.378 & 0.047 \\
\hline VCC 1779 & 21.086 & 0.038 \\
\hline VCC 1895 & 13.803 & 0.022 \\
\hline VCC 1499 & 139.315 & 0.612 \\
\hline VCC 1545 & 41.686 & 0.264 \\
\hline VCC 1192 & 194.088 & 1.841 \\
\hline VCC 1857 & 15.703 & 0.098 \\
\hline VCC 1075 & 26.546 & 0.066 \\
\hline VCC 1948 & 16.143 & 0.161 \\
\hline VCC 1627 & 58.076 & 0.129 \\
\hline VCC 1440 & 34.355 & 0.043 \\
\hline VCC 230 & 101.859 & 0.071 \\
\hline VCC 2050 & 172.186 & 0.197 \\
\hline VCC 1993 & 172.186 & 0.125 \\
\hline VCC 751 & 155.596 & 0.043 \\
\hline VCC 1828 & 58.613 & 0.124 \\
\hline VCC 538 & 152.756 & 0.195 \\
\hline VCC 1407 & 244.343 & 0.664 \\
\hline VCC 1886 & 188.799 & 0.237 \\
\hline VCC 1199 & 296.483 & 1.346 \\
\hline VCC 1743 & 16.443 & 0.032 \\
\hline VCC 1539 & 285.759 & 0.509 \\
\hline VCC 1185 & 285.759 & 0.854 \\
\hline VCC 1826 & 27.289 & 0.031 \\
\hline VCC 1512 & 22.698 & 0.239 \\
\hline VCC 1489 & 76.559 & 0.787 \\
\hline VCC 1661 & 31.332 & 0.064 \\
\hline
\end{tabular}

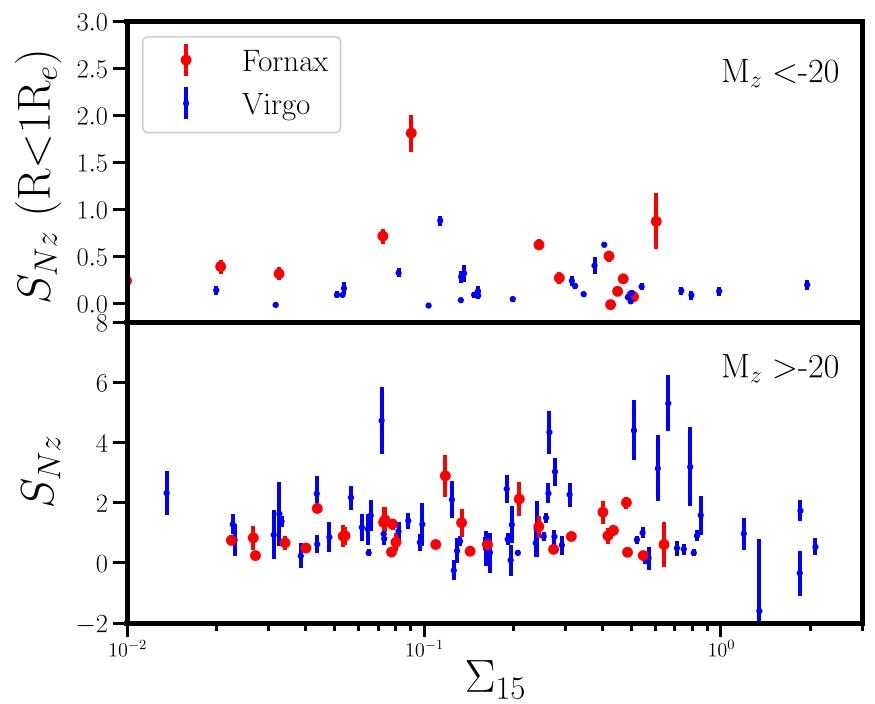

Figure 3. Upper: $S_{N, z}$ of the inner $1 R_{e}$ of massive ETGs and their relation with environmental density $\Sigma_{15}$. There is no clear environmental dependence of inner $S_{N, z}$. Lower: relationship between the $S_{N, z}$ of low-mass ETGs and $\Sigma_{15}$. A fraction of $S_{N, z}$ increases in very dense regions, but $S_{N, z}$ is around zero in the densest environments.

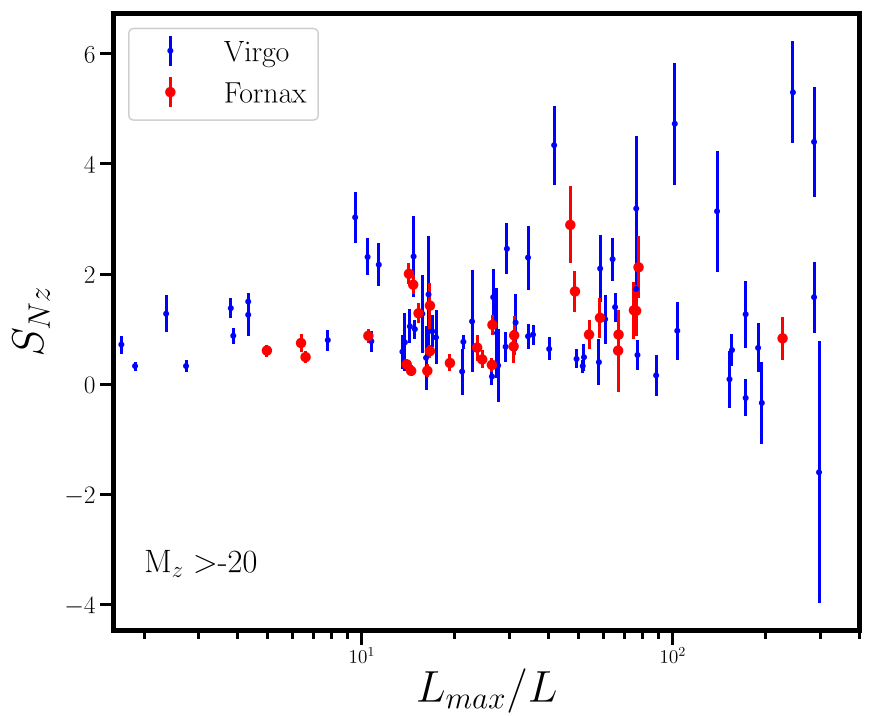

Figure 4. Relationship between the $S_{N, z}$ of low-mass ETGs and $L_{\max } / L$ in the $B$ band. The latter indicates the strength of the tidal field. Here $S_{N, z}$ is roughly constant around 1.5 below $L_{\max } / L \sim 10$. At higher $L_{\max } / L$, the scatter increases with $L_{\max } / L$.

$S_{N, z}$ values of low-mass ETGs are dispersed over the full range of $\Sigma_{15}$ with a scatter larger than that of the intermediate-mass galaxies. The scatter significantly increases in very dense regions. In the very densest environments, however, all of the low-mass ETGs have $S_{N, z}$ close to or consistent with zero. We propose that this is possibly due to tidal stripping from nearby massive galaxies.

In Figure 4, we plot the $S_{N, z}$ of low-mass ETGs against $L_{\max } / L$. Below $L_{\max } / L \sim 10, S_{N, z}$ has a constant value around 1.5. At higher $L_{\max } / L$, the scatter increases with $L_{\max } / L$. These are galaxies that have a much more massive neighbor and indicate that the low-mass galaxies in denser environments could either have higher GC formation efficiencies or be significantly underpopulated with GCs (through inhibition or stripping).

The strength of the tidal field a galaxy experiences is determined by not only the $L_{\max } / L$ but also the distance between galaxies. In Figure 5, we plot the $S_{N, z}$ of low-mass ETGs against $R_{P\left(L_{\max }\right)}$, the projected distance from the most luminous galaxy among the 15 closest neighbors (i.e., the galaxy with $L_{\max }$ ), and the distances are normalized by the $R_{e}$ of the galaxy with $L_{\max }$. Circles and triangles represent galaxies from Fornax and Virgo, respectively. The colors indicate the luminosity of their most luminous neighbors (i.e., $L_{\max }$ ), where the darker colors show the higher $L_{\max }$. The galaxies that have FCC 213 or M87 as their most luminous neighbors have black outlines.

All of the low-mass ETGs located closer than $10 R_{e}$ to their most luminous neighbors have low $S_{N, z}$. Similarly, all of the low-mass ETGs that have the most luminous galaxies in our sample as their most luminous neighbors have low $S_{N, z}$. Both suggest that the GC systems of these low-mass ETGs experienced tidal stripping. For the rest of the low-mass ETGs, at each distance, in general, the ones with the highest $S_{N, z}$ have the most massive neighbors. In addition, their $S_{N, z}$, on average, decreases with the normalized distances. These indicate that apart from tidal stripping, a denser environment can also enhance the GC formation efficiency. 


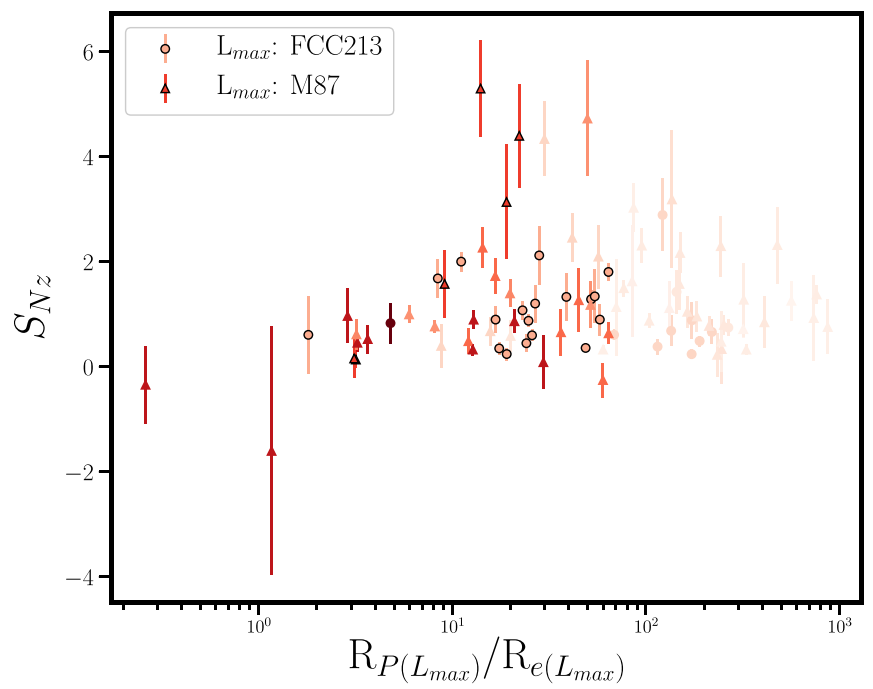

Figure 5. Relationship between $S_{N, z}$ of low-mass ETGs and their distance to the most luminous galaxy among the 15 closest neighbors (i.e., the galaxy with $L_{\max }$ ). The distances are normalized by the $R_{e}$ of the galaxy with $L_{\max }$. Circles and triangles represent galaxies from Fornax and Virgo, respectively. The colors represent the $L_{\max }$, with the darker colors indicating higher $L_{\max }$. The galaxies that have FCC 213 or M87 as their most luminous neighbors have black outlines.

\section{Discussion}

\subsection{Dependence on Galactic Halo Mass}

The variation of GC specific frequency has been shown to follow that of the stellar mass-to-light ratio in galaxies-i.e., the kind of galaxies that have high $S_{N}$ (low- and high-mass) are also the kind that have high total $M / L$. This idea has been explored by associating galaxies with dark matter halos through abundance matching (i.e., Peng et al. 2008), weak lensing (Hudson et al. 2014), or dynamical mass estimates (Harris et al. 2013; Forbes et al. 2018).

Hudson et al. (2014) studied GC mass fractions across a wide mass range. They found that the mass ratio of GCs to the halos of their host galaxies, $\eta$, is constant below a stellar mass $\mathcal{M}_{\star}=10^{11} M_{\odot}$ or halo mass $\mathcal{M}_{h}=10^{13} M_{\odot}$ and slightly decreases toward the higher masses. However, the GC numbers of Fornax galaxies in their sample were adopted from Harris et al. (2013), which are less accurate than what we present (see Section 3.1). Here we investigate this relationship with our improved measurements and more homogeneous data set.

We divide the GCs into blue and red populations in each galaxy and convert the total luminosity of these two populations into stellar masses by their mean colors using the 12 Gyr single stellar population model of Bruzual \& Charlot (2003). The total GC masses are the sum of these two populations. Note that the assumption of $12 \mathrm{Gyr}$ is not suitable for FCC 21, which contains a substantial number of intermediate-age red GCs. To estimate the lower-mass limit, we estimate the total mass of its GC system by assuming that all of its red GCs have an age of 3 Gyr. In this case, the total GC mass in FCC 21 decreases by $25 \%$. Because the change is within the uncertainty, we do not show this lower-mass limit in the plot.

Galactic halo masses are inferred from the galactic stellar masses according to Equation (21) in Behroozi et al. (2010) in the local universe. We use the scale factor for today $(a=1)$

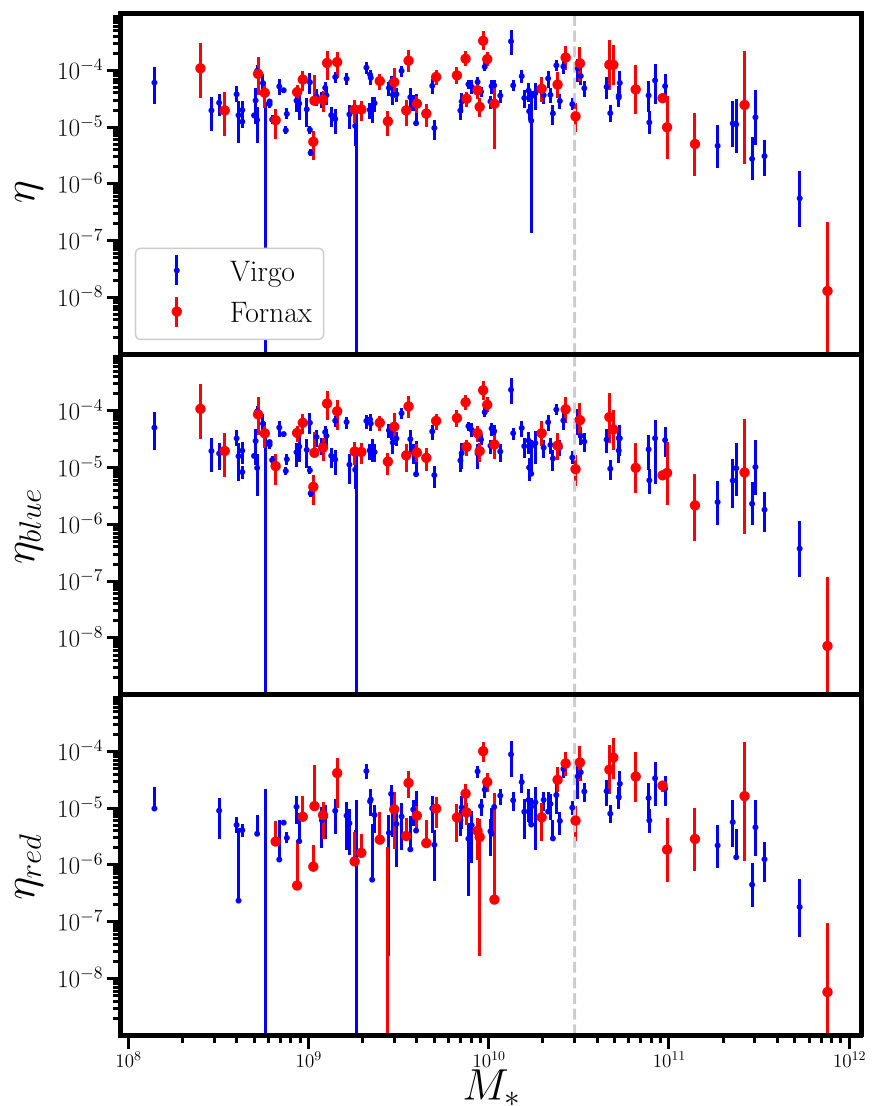

Figure 6. Galactic mass dependence of $\eta$ (the ratio of GC mass to galactic halo mass). The top, middle, and bottom panels show the $\eta$ distributions of the total, blue GC, and red GC populations, respectively. Red circles and blue dots represent the ETGs from Fornax and Virgo, respectively. For clarity, we do not plot the lower error bars, which are larger than the measurements themselves, but we use them in our analysis. In all panels, the GC mass fraction of ETGs more massive than $3 \times 10^{10} M_{\odot}$ stellar mass (dashed lines) decreases toward the high-mass end. Among the galaxies that are less massive, the $\eta$ values of blue and total GCs are generally constant, but that of red GCs slightly increases with mass.

and adopt the best-fitting parameters at $\mu=\kappa=0$. Like in other work, these halo masses are thus not direct measurements but are inferred from a global stellar mass-halo mass relation.

Figure 6 displays how $\eta$ distributes with galactic stellar mass. From top to bottom, $\eta$ is calculated for the total GC population, blue GCs, and red GCs, respectively. Red circles and blue dots represent the ETGs from Fornax and Virgo, respectively. We only plot the data points with positive numbers of GCs but point out that the existence of galaxies with zero GCs (but, presumably, with a nonzero halo mass) shows that the relationship must break down at some point or has large scatter.

In all panels, the GC mass fraction decreases toward the high-mass end when $M_{*}$ exceeds $3 \times 10^{10} M_{\odot}$ (dashed lines), reproducing the trends in Hudson et al. (2014). A possible reason is that the halo masses estimated by Behroozi et al. (2010) are high in this range. El-Badry et al. (2019) investigated this relation between GC masses and halo masses using a semi-analytic model built on dark matter merger trees. They produced a linear relation for massive galaxies, and the halo masses in their simulation are lower than what Behroozi et al. (2010) predicted at given stellar masses (see Figure 13 in 
El-Badry et al. 2019). It is also the case that the stellar masshalo mass relation becomes very steep at these stellar masses, so determining $\eta$ for these massive galaxies becomes problematic.

Among the galaxies with lower masses, the $\eta$ values of blue and total GCs are generally constant. On the other hand, the mass fraction of red GCs increases slightly with mass, indicating a lower formation efficiency of metal-rich GCs in lower-mass halos. El-Badry et al. (2019) produced similar relations in their simulation. In addition, they found that the linear relation at the massive end is a result of the central limit theorem during the hierarchical assembly, independent of GC formation scenarios. However, for galaxies with lower masses, the physics of GC formation is required to reproduce the relations.

If the $\mathrm{GC}$ formation efficiency with respect to halo masses is universal, as suggested by the constant mean $\eta$ at low masses, the intrinsic scatter of $\eta$ reflects the variation of field star formation efficiency, i.e., the scatter in $M_{h} / L$. For the low-mass ETGs with $M_{z}>-20$, we modify MPFITEXY (Press et al. 1992; Tremaine et al. 2002), allowing for fitting with asymmetric errors, and fit their zero-slope linear relation between $\log (\eta)$ and $\log \left(\mathcal{M}_{\star}\right)$. We perform 5000 bootstrap iterations on each fit to calculate the errors of our fitting parameters. The results show that they have a mean $\eta$ of $(3.36 \pm 0.25) \times 10^{-5}$ with an intrinsic scatter of $0.30 \pm 0.04$ dex, in agreement with the results from other studies (Georgiev et al. 2010; Hudson et al. 2014).

In our analysis, we do not include the uncertainty of the galactic stellar mass-halo mass relation. The simulations from Behroozi et al. (2010) showed a typical scatter of 0.15-0.2 dex, which is not able to fully account for the intrinsic scatter in our results. It implies that the GC mass fraction has scatter about 0.2 dex. Alternatively, the simulation underestimated the scatter of the $\mathcal{M}_{\star}-\mathcal{M}_{h}$ relation, and the variation of $\eta$ is smaller.

\subsection{GCs and the Origin of Nuclear Star Clusters}

The timescales for GCs to sink into the centers of low-mass ETGs by dynamical friction can be less than the Hubble time. Tremaine et al. (1975) suggested that galactic nuclei formed by the mergers of the GCs fallen into centers. Lotz et al. (2001) examined this hypothesis by comparing the central deficiency of the GC radial density profiles and the brightness of their nuclei, and they found that the nuclei are brighter than expected. This could be a result of subsequent star formation in the galactic center, as Lotz et al. (2004) found that some nuclei in low-mass ETGs experienced recent star formation. It is also possible that some GCs had been disrupted into field stars during their inspiral, because the tidal force is increasing toward the galactic center.

Here we explore the link between GCs and nuclei, comparing the GC numbers with the properties of stellar nuclei. The upper panel of Figure 7 displays the relation between the number of GCs and the $M_{z}$ of the nuclei (Côté et al. 2006; Blakeslee et al. 2009; Turner et al. 2012) in their hosts among the nucleated ETGs. Red and blue symbols represent the galaxies from Fornax and Virgo. Open squares and filled circles represent massive and low-mass ETGs, respectively. The GC number is higher when the hosts have brighter nuclei, which can be a result of two positive
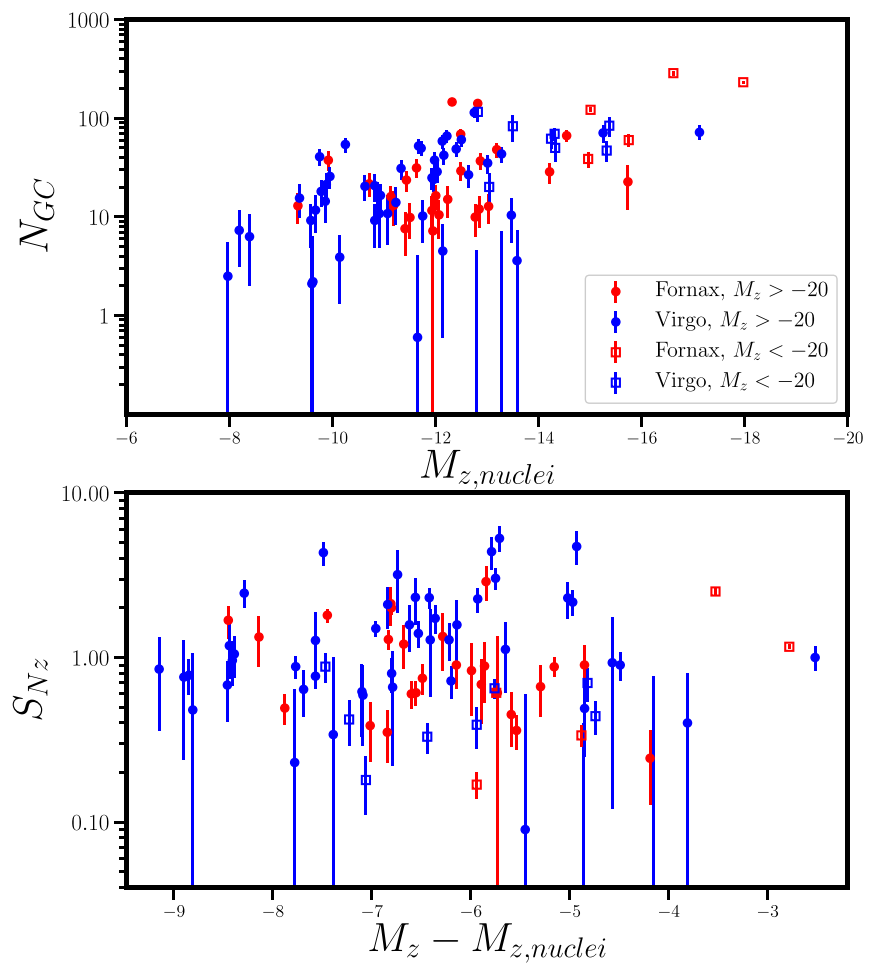

Figure 7. Upper: relation between the number of GCs and the $M_{z}$ of the nuclei of their hosts among the nucleated ETGs. Red and blue symbols represent the galaxies from Fornax and Virgo. Open squares and filled circles represent massive and low-mass ETGs, respectively. The GC number is higher in the hosts with brighter nuclei. Lower: relation between $S_{N, z}$ and the magnitude difference between the nucleated galaxies and their nuclei, which are the normalization of the GC numbers and nuclear light by the luminosity of galactic field stars. These two parameters are not clearly correlated, indicating a weak link between the infall of GCs and nuclear formation.

correlations. One is between the number of GCs and the mass of their host galaxies. The other is between the galactic luminosity and the nuclear luminosity (Lotz et al. 2004; Côté et al. 2006).

However, when normalizing them to the galactic luminosity (lower panel of Figure 7), $S_{N, z}$ shows no dependence on the magnitude difference between the galaxies and their nuclei ( i.e., the nuclear luminosity fraction). If there is a constant fraction of stars that end up in GCs during early star formation, and then the nuclei are built up due to a fraction of GCs that sink into the center, we should expect an anticorrelation between $S_{N, z}$ and $M_{z}-M_{z \text {,nuclei }}$ (i.e., larger nuclei mean fewer remaining GCs). Even if a component of the nuclei is made of subsequent star formation, or some GCs are disrupted before sinking in, this anticorrelation might still be evident if the GC inspiral is the dominant mechanism. It appears that not all galactic nuclei are made from the GCs that spiraled into the centers. This is in agreement with the recent study of the stellar populations of galactic nuclei in the ACSVCS galaxies (Spengler et al. 2017).

\section{Summary}

We measure GC specific frequencies $\left(S_{N}, S_{N, z}\right)$ in 43 galaxies in the Fornax Cluster, a complete sample of Fornax ETGs brighter than $M_{B} \sim-16$ mag. Together with the homogeneous measurements of 100 ETGs in Virgo from Peng et al. (2008), 
we study the GC formation efficiency in ETGs over a wide range of galactic mass and environmental density.

1. The $S_{N, z}$ of ETGs has similar properties in Fornax and Virgo. The 1 order of magnitude difference in the densities of these two galaxy clusters does not have a significant effect on GC formation in ETGs.

2. The $S_{N, z}$ of ETGs in both galaxy clusters has similar distributions with galactic $z$-band absolute magnitude, an indicator of galactic stellar mass. Here $S_{N, z} \sim 1.5$ for intermediate-mass ETGs and increases with $M_{z}$ at the bright end $\left(M_{z}<-23 \mathrm{mag}\right)$. In the low-mass range $\left(M_{z}>-20 \mathrm{mag}\right)$, it also rises, on average, but with large scatter.

3. The $S_{N, z}$ of low-mass ETGs has an environmental dependence. (1) Low-mass ETGs that are located within $10 R_{e}$ of their massive host galaxies or have the most luminous galaxies in our sample as their neighbors have universally low $S_{N, z}$, showing that their GC systems have likely been tidally stripped. (2) For the remaining lowmass ETGs, being closer to their massive neighbors (but not too close) results in them having higher $S_{N, z}$. At a given distance from their massive neighbors, the ones with higher $S_{N, z}$ generally have more massive neighbors. These indicate that apart from tidal stripping, a denser environment can also enhance the GC formation efficiency.

4. The mass ratio between GC systems and the halos of their host galaxies is constant with an intrinsic scatter of $\sim 0.3 \mathrm{dex}$ when galactic stellar masses are below $3 \times 10^{10} M_{\odot}$ (corresponding to a halo mass of $3 \times$ $10^{12} M_{\odot}$ ). It slightly decreases toward the massive end in the higher-mass range, where inferring halo masses from stellar masses becomes difficult. These are in agreement with previous studies. The constant GC mass fraction of low-mass ETGs $\left(\eta \sim 3.36 \times 10^{-5}\right.$, on average) suggests a uniform formation efficiency of GCs with respect to the dark matter halo masses and that the GC formation capability is fundamentally governed by the potential wells of galaxies.

Y.L. acknowledges support from the Oxford Centre for Astrophysical Surveys, which is funded through generous support from the Hintze Family Charitable Foundation. She also thanks Shuo Yuan and Long Wang for the helpful discussions on statistics. Y.L. and E.W.P. acknowledge support from the National Natural Science Foundation of China under grant No. 11573002 and the Strategic Priority Research Program, "The Emergence of Cosmological Structures," of the Chinese Academy of Sciences, grant No. XDB09000105. T.H.P. acknowledges support through FONDECYT Regular project 1161817 and CONICYT project Basal AFB-170002.

\section{ORCID iDs}

Yiqing Liu (1) https://orcid.org/0000-0002-4789-2195

Eric W. Peng (i) https://orcid.org/0000-0002-2073-2781

Andrés Jordán (1) https://orcid.org/0000-0002-5389-3944

John P. Blakeslee (1) https://orcid.org/0000-0002-5213-3548

Patrick Côté (i) https://orcid.org/0000-0003-1184-8114

Laura Ferrarese (1) https://orcid.org/0000-0002-8224-1128

Thomas H. Puzia (ib https://orcid.org/0000-0003-0350-7061

\section{References}

Bassino, L. P., Richtler, T., \& Dirsch, B. 2006, MNRAS, 367, 156 Baum, W. A. 1955, PASP, 67, 328

Behroozi, P. S., Conroy, C., \& Wechsler, R. H. 2010, ApJ, 717, 379

Blakeslee, J. P. 1997, ApJL, 481, L59

Blakeslee, J. P. 1999, AJ, 118, 1506

Blakeslee, J. P., Jordán, A., Mei, S., et al. 2009, ApJ, 694, 556

Blakeslee, J. P., Tonry, J. L., \& Metzger, M. R. 1997, AJ, 114, 482

Brodie, J. P., \& Strader, J. 2006, ARA\&A, 44, 193

Bruzual, G., \& Charlot, S. 2003, MNRAS, 344, 1000

Côté, P., Blakeslee, J. P., Ferrarese, L., et al. 2004, ApJS, 153, 223

Côté, P., Marzke, R. O., \& West, M. J. 1998, ApJ, 501, 554

Côté, P., Piatek, S., Ferrarese, L., et al. 2006, ApJS, 165, 57

Dirsch, B., Richtler, T., Geisler, D., et al. 2003, AJ, 125, 1908

El-Badry, K., Quataert, E., Weisz, D. R., Choksi, N., \& Boylan-Kolchin, M. 2019, MNRAS, 482, 4528

Elmegreen, B. G., \& Efremov, Y. N. 1997, ApJ, 480, 235

Ferguson, H. C. 1989, AJ, 98, 367

Ferrarese, L., Côté, P., Jordán, A., et al. 2006, ApJS, 164, 334

Forbes, D. A., Read, J. I., Gieles, M., \& Collins, M. L. M. 2018, MNRAS, 481,5592

Ford, H. C., Bartko, F., Bely, P. Y., et al. 1998, Proc. SPIE, 3356, 234

Georgiev, I. Y., Puzia, T. H., Goudfrooij, P., \& Hilker, M. 2010, MNRAS, 406, 1967

Goudfrooij, P., Alonso, M. V., Maraston, C., \& Minniti, D. 2001a, MNRAS, 328,237

Goudfrooij, P., Gilmore, D., Whitmore, B. C., \& Schweizer, F. 2004, ApJL, 613, L121

Goudfrooij, P., Mack, J., Kissler-Patig, M., Meylan, G., \& Minniti, D. 2001b, MNRAS, 322, 643

Guérou, A., Emsellem, E., McDermid, R. M., et al. 2015, ApJ, 804, 70

Harris, W. E. 1991, ARA\&A, 29, 543

Harris, W. E., Harris, G. L. H., \& Alessi, M. 2013, ApJ, 772, 82

Harris, W. E., \& van den Bergh, S. 1981, AJ, 86, 1627

Hudson, M. J., Harris, G. L., \& Harris, W. E. 2014, ApJL, 787, L5

Jordán, A., Blakeslee, J. P., Côté, P., et al. 2007a, ApJS, 169, 213

Jordán, A., Blakeslee, J. P., Peng, E. W., et al. 2004, ApJS, 154, 509

Jordán, A., Côté, P., Blakeslee, J. P., et al. 2005, ApJ, 634, 1002

Jordán, A., McLaughlin, D. E., Côté, P., et al. 2007b, ApJS, 171, 101

Jordán, A., Peng, E. W., Blakeslee, J. P., et al. 2009, ApJS, 180, 54

Jordán, A., Peng, E. W., Blakeslee, J. P., et al. 2015, ApJS, 221, 13

Kimm, T., Cen, R., Rosdahl, J., \& Yi, S. K. 2016, ApJ, 823, 52

Kravtsov, A. V., \& Gnedin, O. Y. 2005, ApJ, 623, 650

Kruijssen, J. M. D. 2012, MNRAS, 426, 3008

Larsen, S. S., Brodie, J. P., Huchra, J. P., Forbes, D. A., \& Grillmair, C. J. 2001, AJ, 121, 2974

Larsen, S. S., \& Richtler, T. 2000, A\&A, 354, 836

Liu, Y., Ho, L. C., \& Peng, E. 2016a, ApJL, 829, L26

Liu, Y., Peng, E. W., Blakeslee, J., et al. 2016b, ApJ, 818, 179

Liu, Y., Peng, E. W., Lim, S., et al. 2016c, ApJ, 830, 99

Lotz, J. M., Miller, B. W., \& Ferguson, H. C. 2004, ApJ, 613, 262

Lotz, J. M., Telford, R., Ferguson, H. C., et al. 2001, ApJ, 552, 572

Masters, K. L., Jordán, A., Côté, P., et al. 2010, ApJ, 715, 1419

McLaughlin, D. E. 1999, AJ, 117, 2398

Miller, B. W., \& Lotz, J. M. 2007, ApJ, 670, 1074

Mistani, P. A., Sales, L. V., Pillepich, A., et al. 2016, MNRAS, 455, 2323

Peng, E. W., Jordán, A., Côté, P., et al. 2006, ApJ, 639, 95

Peng, E. W., Jordán, A., Côté, P., et al. 2008, ApJ, 681, 197

Press, W. H., Teukolsky, S. A., Vetterling, W. T., \& Flannery, B. P. 1992 , Numerical Recipes in FORTRAN. The Art of Scientific Computing (Cambridge: Cambridge Univ. Press)

Richtler, T., Hilker, M., Kumar, B., et al. 2014, A\&A, 569, A41

Spengler, C., Côté, P., Roediger, J., et al. 2017, ApJ, 849, 55

Spitler, L. R., \& Forbes, D. A. 2009, MNRAS, 392, L1

Tonini, C. 2013, ApJ, 762, 39

Tremaine, S., Gebhardt, K., Bender, R., et al. 2002, ApJ, 574, 740

Tremaine, S. D., Ostriker, J. P., \& Spitzer, L., Jr. 1975, ApJ, 196, 407

Turner, M. L., Côté, P., Ferrarese, L., et al. 2012, ApJS, 203, 5

Villegas, D., Jordán, A., Peng, E. W., et al. 2010, ApJ, 717, 603

Whitmore, B. C., Zhang, Q., Leitherer, C., et al. 1999, AJ, 118, 1551

Wolf, J., Martinez, G. D., Bullock, J. S., et al. 2010, MNRAS, 406, 1220

Zaritsky, D., Aravena, M., Athanassoula, E., et al. 2015, ApJ, 799, 159

Zepf, S. E., \& Ashman, K. M. 1993, MNRAS, 264, 611 